\title{
LISTADO DE PLANTAS ENDÉMICAS Y EN RIESGO DE LA RESERVA de la Biosfera El Triunfo, Chiapas, MÉXico
}

\author{
Rubén Martínez-Camilo', Miguel Ángel Pérez-Farrera y Nayely Martínez-Meléndez \\ Herbario Eizi Matuda, Facultad de Ciencias Biológicas, Universidad de Ciencias y Artes de Chiapas, \\ Tuxtla Gutiérrez, México \\ 'Autor para la correspondencia: ruben.martinez@unicach.mx
}

\begin{abstract}
Resumen: Se presenta una revisión de las especies de plantas vasculares y no vasculares endémicas y en riesgo de la Reserva de la Biosfera El Triunfo. A partir de un listado del inventario florístico que incluye 2,990 especies y 12,835 registros de herbario, se revisó la inclusión de especies en la Norma Oficial Mexicana (NOM-059-SEMARNAT-2010) y la Lista Roja de la Unión Internacional para la Conservación de la Naturaleza y se determinó su nivel de endemismo de acuerdo con su distribución geográfica. Se obtuvo un listado con 347 especies endémicas, de los cuales: $5.9 \%$ son endémicas a la reserva, $23.9 \%$ a Chiapas y $27.6 \%$ a Chiapas y Guatemala. De las 244 especies listadas en alguna categoría de riesgo, la norma oficial Mexicana refiere: 28 sujetas a protección, 36 amenazadas y 11 en peligro de extinción. La Lista Roja de la Unión Internacional para la Conservación de la Naturaleza reconoce: 29 especies casi amenazadas, 74 vulnerables, 63 en peligro y 16 en peligro crítico. El número de especies endémica y en riesgo representan $17 \%$ de la flora de la reserva y $4.2 \%$ de la flora de Chiapas. El bosque mesófilo de montaña fue el ecosistema con mayor número de especies en riesgo y endémicas. La Reserva de la Biosfera El Triunfo tiene una gran biodiversidad y protege un importante número de especies endémicas y en riesgo.
\end{abstract}

Palabras claves: área natural protegida, base de datos, conservación, riqueza florística, Sierra Madre de Chiapas.

\begin{abstract}
This paper is a review of the endemic and threatened plant species of El Triunfo Biosphere Reserve. From a floristic inventory that includes 2,990 species and 12,835 herbarium records, we reviewed the presence of each species in the official listings of the Mexican Norm (NOM-059-SEMARNAT-2010) and Red List of International Union for Conservation of Nature, determined its level of endemism with support of a database. We obtained a checklist of 347 endemic species: $5.9 \%$ are endemic to the El Triunfo Reserve, $23.9 \%$ to Chiapas and $27.6 \%$ to Chiapas and Guatemala. We found 244 species at risk, for Mexican Norm status are: 28 to be protected, 36 threatened, and 11 endangered. For International Union for Conservation of Nature Red List categories are: 16 critically endangered, 63 endangered, 74 vulnerable, and 29 near threatened. The number of species endemics and threatened represent $17 \%$ of the estimated flora for El Triunfo Reserve and $4.2 \%$ of the flora of Chiapas. The cloud forest ecosystem presented the highest number of threatened and endemic species. The El Triunfo Reserve is located in a floristically diverse geographic region and protects a significant number of plant species both endemic and threatened.
\end{abstract}

Keywords: conservation, database, floristic richness, natural protected area, Sierra Madre de Chiapas.

L a Reserva de la Biosfera El Triunfo (REBITRI, en adelante) se localiza en la porción central de la región fisiográfica Sierra Madre de Chiapas; se trata de una de las áreas naturales protegidas más importantes de México dado que contiene uno de los relictos más extensos y mejor conservados de bosque mesófilo de montaña (sensu Rzedowski, 1978; Challenger, 1998). Tiene una gran riqueza de fauna (Espinosa-Medinilla et al., 1998) y flora (Long y Heath, 1991; SEMARNAP, 1998). Parte de la REBITRI se encuentra dentro de una región considerada como uno de los refugios primarios del Pleistoceno, razón por la cual es un centro de alta biodiversidad y endemismo (Toledo, 1982).

Pérez-Farrera (2004) señala que la reserva podría albergar alrededor de 3,000 especies de plantas; aproximadamente un $36 \%$ de la estimada por Breedlove (1981) para Chiapas. Algunos antecedentes al conocimiento de la flora dentro de la REBITRI son los de Matuda (1950), quien documentó 791 especies de plantas en el Monte Ovando; Long y Heath (1991), reportan la presencia de $751 \mathrm{spp}$. para el polígono 1; Martínez-Meléndez et al. (2008), indican que 
hay 502 spp. en el Cerro Cebú; Pérez-Farrera et al. (2012), obtuvieron $795 \mathrm{spp}$. para el polígono III y áreas adyacentes y Martínez-Meléndez et al. (2009) documentan 465 especies de epífitas vasculares. Respecto a los tipos de vegetación, en SEMARNAP (1998) se mencionan diez de los 19 tipos de vegetación propuestos por Breedlove (1981) para Chiapas y Pérez-Farrera (2004) señala siete tipos de vegetación usando la nomenclatura de Rzedowski (1978); de éstos destaca por su extensión e importancia el bosque mesófilo de montaña.

El endemismo, es un atributo de la biodiversidad que hace referencia al aislamiento de una especie o grupo de especies a un área geográfica específica, y que no es posible encontrar en ningún otro lado. La designación de una especie como endémica o del nivel de endemismo, depende de la escala geográfica que se considere y del estado de conocimiento de la distribución de la especie. Los límites en la distribución geográfica de una especie, cuando se designa como endémica, pueden obedecer a límites naturales que están definidos por condiciones de tipo insular, fisiográfico, climático y edáfico, por mencionar algunos; en otros casos, los límites pueden ser designados con base en una delimitación de tipo político o geopolítico (Rzedowski, 1991a,b, 1998; Dirzo y Raven, 2003). Rzedowski (1991b, 1998) señala la importancia de conocer el endemismo de una región como una singularidad cuantificable y comparable con otras regiones.

El conocer las especies en riesgo o amenazadas es importante para tomar medidas cautelares para su protección y conservación. En México, se han realizado algunos trabajos que aportan listados o compilaciones de especies de plantas amenazadas, en peligro de extinción o endémicas (Vovides et al., 1997; SEMARNAT, 2002, 2010; González-Espinosa et al., 2011); Vovides et al. (1997) señalan la importancia de listados de plantas de grupos específicos o de regiones geográficas particulares, ya que ofrecen un alto nivel de detalle. Por ejemplo, los realizados para Bromeliaceae (Espejo-Serna et al., 2004), Cactaceae (Hernández y Godínez, 1994), gimnospermas (Contreras-Medina y Luna-Vega, 2007), Heliantheae (Villaseñor, 1991), Magnoliophyta (Villaseñor, 2003), Orchidaceae (Soto-Arenas et al., 2007), Poaceae (Dávila-Aranda et al., 2004) y Ternstroemiaceae (LunaVega et al., 2004). Ejemplos por región, se tienen los de la Mixteca Alta (García-Mendoza et al., 1994), Tehuacán-Cuicatlán (Méndez-Larios et al., 2004) y los estados de Oaxaca (Acosta-Castellanos, 2002) y Veracruz (Castillo-Campos $e t$ al., 2005).

El papel que desempeña un área natural protegida en la conservación de la biodiversidad, toma mayor relevancia cuando se tiene conocimiento de las especies que se encuentran en ella; principalmente de las endémicas, amenazadas, en peligro de extinción u otra categoría de riesgo.

Con base en esta información es posible incidir en políticas de conservación más apropiadas y enfocadas en aquellas especies que lo requieran. Ceballos et al. (2009) mencionan que son pocas las áreas naturales protegidas de México que cuentan con un inventario reciente y confiable, lo que subestima el número de especies restringidas presentes en ellas. El objetivo de este trabajo es presentar un listado de las especies de plantas vasculares de la REBITRI que están incluidas en alguna categoría de riesgo por la Norma Mexicana e internacional, y de aquellas especies con algún nivel de endemismo, según su distribución geográfica conocida.

\section{Materiales y métodos}

Área de estudio. La REBITRI se localiza en la porción sur del estado de Chiapas, México. Específicamente en la Sierra Madre de Chiapas entre los $15^{\circ} 09^{\prime} 10^{\prime \prime}$ y $15^{\circ} 57^{\prime} 02^{\prime \prime}$ latitud norte y $92^{\circ} 34^{\prime} 04$ " y $93^{\circ} 12^{\prime} 42^{\prime \prime}$ longitud oeste. Tiene una superficie de 119,117 ha. Su superficie está zonificada en cinco polígonos o zonas núcleos: Custepec, El Triunfo, El Venado, La Angostura y Ovando y una zona de amortiguamiento. Se caracteriza por tener altitudes que van de 450 a 3,000 m s.n.m. En general, comprende un paisaje montano de estructuras plegadas que corren en dirección NO-SE y sirve de parteaguas entre la vertiente del Pacífico y la Depresión Central de Chiapas (SEMARNAP, 1998).

La REBITRI se encuentra en una zona de transición entre la región neartica y la neotropical. Se localiza entre la unidad orogénica de la Sierra Madre de Chiapas y la región geomórfica Planicie Costera Ístmica-Chiapaneca. Las formaciones geológicas presentes son del Precámbrico y Paleozoico (complejo basal), también existen estratos del Mesozoico y Cenozoico (Müllerried, 1957). Existen cuatro tipos climáticos: (1) cálido subhúmedo, con lluvias en verano, se encuentra al noroeste de la REBITRI de ambas vertientes y hasta una altitud de 1,000 m s.n.m., (2) semicálido húmedo, con abundantes lluvias en verano, se encuentra entre los 1,000 y los 2,000 m de ambas vertientes, (3) cálido húmedo, con abundantes lluvias en verano, se encuentra en las partes bajas de la vertiente del Pacífico hasta una altura de 1,000 m y (4) templado húmedo, con abundantes lluvias en verano, se encuentra al este de la reserva, en alturas superiores a los 2,000 $\mathrm{m}$ de ambas vertientes, en todos los municipios excepto el de Villa Corzo (SEMARNAP, 1998). Se mencionan siete tipos de vegetación para la REBITRI según la clasificación de Rzedowski (1978): bosque de coníferas, de Quercus, mesófilo de montaña, tropical caducifolio, tropical perennifolio, tropical subcaducifolio y vegetación acuática y subacuática (Pérez-Farrera, 2004). Destacan por su extensión, en orden de importancia, el bosque mesófilo de montaña, el tropical perennifolio y de coníferas. Long y Heath (1991) describen siete asociaciones vegetales de bosque mesófilo tan sólo para el polígono 1 de la REBITRI, éstos son: Gaultheria-Ugni-Vaccinium, Quercus-Matudaea-Hedyosmum-Dendropanax, Liquidambar-Quercus-Pinus, Cupressus-Pinus, Ficus-Coccoloba-Dipholis-Sapium, Garcinia-Inga-Desmopsis y Quercus salicifolia. 
Método. Se utilizó la base de datos del listado florístico de la REBITRI conformada por 12,835 registros de herbario. El cual incluye 2,990 especies de plantas vasculares y no vasculares (Pérez-Farrera et al., datos no publicados). La base de datos contiene todas las colectas realizadas hasta el momento dentro de los límites de la REBITRI; las primeras fueron realizadas en la década de 1930 (Matuda, 1950). Para cada especie se determinó el estado de conservación revisando su inclusión en alguna categoría de la Norma Oficial Mexicana (SEMARNAT, 2010) y de la Lista Roja de especies amenazadas de la Unión Internacional para la Conservación de la Naturaleza, IUCN por sus siglas en inglés (González-Espinosa et al., 2011; IUCN, 2011).

Para averiguar que especies son endémicas, se revisó la distribución de cada especie mediante la consulta de obras como artículos en revistas especializadas, monografías y floras, destacando las siguientes: El bosque húmedo de montaña en México y sus plantas vasculares (Villaseñor, 2010); Flora de Chiapas (Breedlove, 1981), Flora Mesoamericana (Davidse et al., 1994, 1995, 2009), Flora Neotropical, Eupatorieae de México (Turner, 1997), Pteridoflora de México (Mickel y Smith, 2004) y Rubiáceas de México (Borhidi, 2006). También se consultaron las bases de datos del Jardín Botánico de Missouri <www.tropics.org> y de la Academia de Ciencias de California <www.research.calacademy.org $>$. Para asignarle a una especie de planta alguna categoría de endemismo, se consideró que su distribución estuviera restringida a la REBITRI, a Chiapas o en combinación de Chiapas con otros estados de México y/o países de Centroamérica. La presencia de una especie en cuatro o más estados de México o en combinación con los países de Centroamérica aumentaba, relativamente, su nivel de distribución más allá del objetivo del presente trabajo, por lo que éstas no se consideraron. La designación en este trabajo del endemismo a un marco geográfico-político es discutible, principalmente por la ausencia de una base biológica y el uso arbitrario de límites políticos. Sin embargo, se optó por esta forma arbitraria valorando la dificultad de establecer límites regionales naturales. De acuerdo con lo anterior, se tomaron en cuenta los estados de México colindantes con Chiapas (Oaxaca, Tabasco y Veracruz), el estado de Guerrero (no se encontraron especies con presencia en Chiapas en combinación con otros estados de México, por ejemplo Campeche) y los países de Centroamérica (Belice, Costa Rica, El Salvador, Guatemala, Honduras, Nicaragua y Panamá).

Para cada especie seleccionada se revisó su hábito, para lo cual se definieron seis formas: (1) árbol, planta perenne con tallo lignificado, ramificado por arriba de la base, de más de $3 \mathrm{~m}$ de altura, (2) arborescente, que llega a tener el aspecto de un árbol, a menudo referido a las especies de palmas y helechos grandes, (3) arbusto, planta perenne con tallo lignificado, ramifica a partir de la base y de menos de 3 $m$ de altura, (4) hierba terrestre, con poco tejido leñoso, de baja estatura, (5) hierba epífita, que crece sobre otra planta
Cuadro 1. Distribución cuantitativa por familias, géneros y especies endémicas y en riesgo de la Reserva de la Biosfera El Triunfo, Chiapas, México. $\mathrm{E}=$ endémica, $\mathrm{R}=$ en riesgo.

\begin{tabular}{lllllll}
\hline Grupo & \multicolumn{3}{l}{ Familias } & \multicolumn{2}{c}{ Géneros } & \multicolumn{2}{c}{ Especies } \\
\cline { 2 - 8 } & $\mathrm{E}$ & $\mathrm{R}$ & $\mathrm{E}$ & $\mathrm{R}$ & $\mathrm{E}$ & $\mathrm{R}$ \\
\hline Pteridophyta & 4 & 6 & 4 & 11 & 7 & 14 \\
Coniferophyta & 0 & 3 & 0 & 3 & 0 & 4 \\
Cycadophyta & 1 & 1 & 2 & 2 & 4 & 4 \\
Magnoliophyta & & & & & & \\
$\quad$ Liliopsida & 11 & 5 & 29 & 19 & 66 & 34 \\
$\quad$ Magnoliopsida & 70 & 54 & 171 & 102 & 270 & 188 \\
Total & 86 & 69 & 206 & 137 & 347 & 244 \\
\hline
\end{tabular}

y (6) bejuco, planta trepadora larga, a menudo leñosa (Moreno, 1984). El tipo de vegetación fue de acuerdo al sistema de clasificación de Rzedowski (1978). El arreglo del listado es de acuerdo al sistema de clasificación de Crabbe et al. (1975) con algunas modificaciones por Mickel y Smith (2004) para el grupo de los helechos y afines; Mabberley (1987) para gimnospermas y con algunas modificaciones Cronquist (1988) para las plantas con flores.

\section{Resultados}

Endemismo. Se obtuvo un listado con 347 especies (adscritas a 86 familias) con algún nivel de endemismo de acuerdo a los límites aquí definidos (Cuadro 1). De este total, 5.9\% son endémicas estrictas a la REBITRI, $23.9 \%$ son de Chiapas, $27.6 \%$ se distribuyen en Chiapas y Guatemala y el resto se distribuyen en combinación con otros estados de México y países de Centroamérica (Figura 1). La frecuencia de especies endémicas por su hábito son: árboles (111), arborescente (7), arbusto (67), hierbas terrestres (82), hierbas epífi-

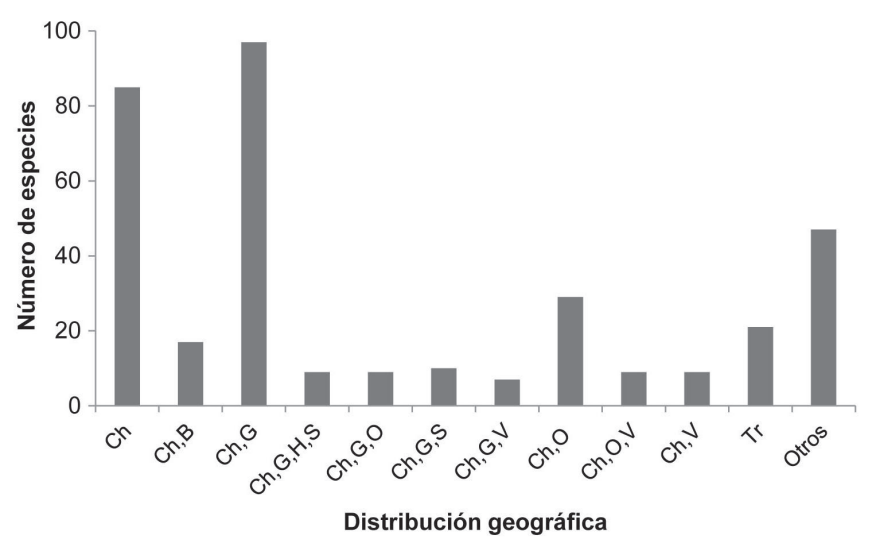

Figura 1. Gráfica del número de especies endémicas y su distribución geográfica en la Reserva de la Biosfera El Triunfo, Chiapas, México. $\mathrm{Ch}=$ Chiapas, $\mathrm{B}=$ Belice, $\mathrm{CR}=$ Costa Rica, $\mathrm{G}=$ Guatemala, $\mathrm{Gu}=$ Guerrero, $\mathrm{H}=$ Honduras, $\mathrm{N}=$ Nicaragua, $\mathrm{O}=$ Oaxaca, $\mathrm{P}=$ Panamá, $\mathrm{Gu}=$ Guerrero, $\mathrm{S}=\mathrm{El}$ Salvador, $\mathrm{T}=$ Tabasco, $\mathrm{Tr}=$ Reserva de la biosfera El Triunfo, $V=$ Veracruz. 
tas (40) y bejucos (40). Las familias con mayor número de especies endémicas fueron Asteraceae (32), Rubiaceae (21), Araceae (15) y Bromeliaceae (13), Fabaceae, Myrsinaceae y Orchidaceae (14 cada una); estas familias agrupan $37 \%$ de las especies. El tipo de vegetación con mayor número de especies endémicas fue el bosque mesófilo de montaña, seguido del tropical perennifolio y de coníferas (Figura 2).

Especies en riesgo. Se reportan 244 especies (en 69 familias) en algún tipo de riesgo (Cuadro 1); 75 están listadas en la NOM-059-SEMARNAT-2010 distribuidos de la siguiente manera (Apéndice): 36 amenazadas, 28 sujetas a protección ambiental y 11 en peligro de extinción. En la Lista Roja de la UICN se encuentran 182 especies, clasificadas en cuatro categorías: 29 aparecen como casi amenazado, 74 vulnerables, 63 en peligro y 16 en peligro crítico. Las familias con mayor número de especies en riesgo son Lauraceae (24 especies), Rubiaceae (20), Myrsinaceae (16), Orchidaceae (15), Fagaceae (13) y Fabaceae (10). De las especies en riesgo, 171 son árboles, 19 arborescentes, 17 arbustos, 28 epífitas y nueve herbáceas terrestres. Los tipos de vegetación más importantes, por la frecuencia de especies en riesgo que en ella se encuentran son: el bosque mesófilo de montaña (192 especies), tropical perennifolio (102) y de coníferas (62, Figura 2).

De las especies en riesgo reportadas en este trabajo, diez son endémicas a México: Beschorneria albiflora (Agavaceae), Ceratozamia vovidesii, Zamia soconuscensis (Zamiaceae), Coccoloba matudae (Polygonaceae), Disocactus macdougallii (Cactaceae), Pleurothallis saccatilabia (Orchidaceae), Maytenus matudae, M. stipitata (Celastraceae), Nectandra matudae (Lauraceae), Quercus benthamii (Fagaceae) y Pseudomiltemia filisepala (Rubiaceae).

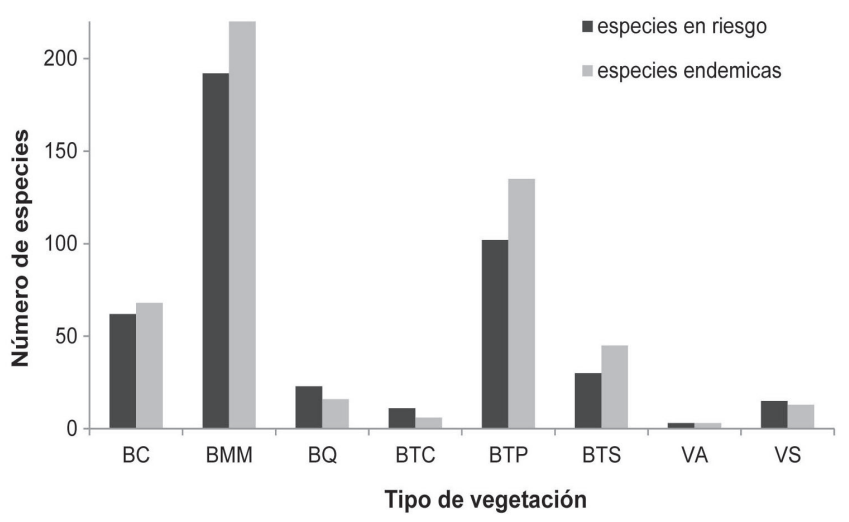

Figura 2. Número de especies amenazadas y en riesgo dentro de cada tipo de vegetación (usando la clasificación de Rzedowski, 1978) presente en la Reserva de la Biosfera El Triunfo, Chiapas. $\mathrm{BC}=$ bosque de coníferas, $\mathrm{BMM}=$ bosque mesófilo de montaña, BQ = bosque de Quercus, BTC = bosque tropical caducifolio, BTP = bosque tropical perennifolio, BTS = bosque tropical subcaducifolio, VA = vegetación acuática y subacuática, VS = vegetación secundaria.
De las especies endémicas, 88 están incluidas en alguna categoría de riesgo, cinco son endémicas a la REBITRI: Arachnothryx pauciflora (Rubiaceae), Buddleja cordata subsp. ovandensis (Loganiaceae), Eugenia ovandensis (Myrtaceae), Ocotea matudae (Lauraceae) y Zamia soconuscencis (Zamiaceae).

\section{Discusión}

El número de especies endémicas encontradas en este trabajo representan aproximadamente $11.6 \%$ de las especies estimadas para la REBITRI (Pérez-Farrera et al., datos no publicados) y $4.2 \%$ de las conocidas para Chiapas (Breedlove, 1981). La familia Asteraceae es la más representada, Villaseñor (1991) describe para la subtribu Heliantheae (Asteraceae) 17 especies restringidas a los límites de Chiapas, de los cuales una especie es endémica para la REBITRI (Schistocarpha matudae). El único género de Asteraceae monotípico y endémico de la REBITRI es Nesomia ( $N$. chiapensis, Turner, 1997).

En Rubiaceae, Borhidi (2006) reporta 15 géneros endémicos de México, de las cuales, dos (Plocaniophyllon y Pseudomiltemia) están presentes en la REBITRI: Pseudomiltemia tiene dos especies que se distribuyen en la REBITRI, una de ellas se encuentra ( $P$. davidsonii) únicamente en esta reserva. Respecto a la familia Orchidaceae, 14 especies presentan algún nivel de endemismo, de las cuales 12 son hierbas epífitas, que representan 7.4\% de las hierbas epífitas de la REBITRI (Martínez-Meléndez et al., 2009); y tres son endémicas de Chiapas: Acianthera breedlovei, Epidendrum erectifolium y E.tacanaense. El número de especies de orquídeas endémicas y en riesgo es importante, considerando que es uno de los grupos con mayor número de especies dentro de la Norma Oficial Mexicana. Soto-Arenas y colaboradores (2007) reconocen 200 especies de orquídeas listadas en la Norma Oficial Mexicana (SEMARNAT, 2002), de las cuales solamente 120 especies tienen poblaciones dentro de un área natural protegida. García-Cruz y Sosa (2011) reportan 18 especies de orquídeas para Veracruz listados en la Norma Oficial Mexicana, un número semejante al encontrado en este trabajo, pero con una diferencia enorme en el tamaño del territorio. También destacan especies de la familia Bromeliaceae, que en Chiapas ocupa el tercer lugar en endemismo con 17 especies (Espejo-Serna et al., 2004), de las cuales cuatro son exclusivas a la REBITRI: Aechmea matudae, Pitcairnia breedlovei, P. matudae y Tillandsia vriesioides.

Algunas especies importantes de la REBITRI, por presentar una distribución geográfica restringida son Abies guatemalensis (Pinaceae) y Zamia soconuscensis (Zamiaceae) (Contreras-Medina y Luna-Vega, 2007); también se encuentran especies muy raras en su distribución, conocidas sólo de unas cuantas localidades, por ejemplo Anthurium sarukhanianum (Araceae), Eupatorium heathiae (Asteraceae, Turner, 1997), Pseudomiltemia filisepala (Borhidi et 
al., 2004), P. davidsonii (Rubiaceae, Martínez-Camilo et al., 2011), Lepanthes matudana (Orchidaceae), Neomortonia nummularia (Gesneriaceae) y Schismocarpus matudae (Loasaceae; Martínez-Meléndez et al., 2009).

La proporción de especies endémicas de Chiapas es similar al encontrado para Chiapas y Guatemala. Lo cual era de esperarse debido a la mayor afinidad fitogeográfica de Chiapas con Centroamérica, particularmente con Guatemala (Rzedowski, 1978; Davidse et al., 1995; Knapp y Davidse, 2006). Esta afinidad es menor en comparación con los estados de Oaxaca, Tabasco y Veracruz. Por ejemplo, la flora de Oaxaca, en estudios de paleoflora se encontró una mayor similitud de elementos florísticos con las floras del centro del país (Puebla) que con Chiapas (Graham, 1998). Rzedowski y Calderón (1989) mencionan que 5\% de las especies de las montañas de Chiapas tienen una distribución restringida, que en ocasiones se extienden a regiones adyacentes con Guatemala. Breedlove (1981) reconoce áreas de endemismo en la Altiplanicie Central, Depresión Central, Montañas del Norte y Sierra Madre de Chiapas.

Según la NOM-ECOL-SEMARNAT-2010, las familias de plantas con mayor número de especies en riesgo, en orden de importancia son Orchidaceae, Arecaceae, Bromeliaceae y Cyatheaceae. Para la Lista Roja de la IUCN (González-Espinosa et al., 2011), las más importantes por el número de especies, son las familias Lauraceae, Rubiaceae y Myrsinaceae. De las 545 especies de plantas incluidas en este tratado, 25.8\% de ellas se encuentran en la REBITRI. Es decir, una de cada cuatro especies en alguna categoría de riesgo que habitan en los ecosistemas de montaña de México se encuentra en la REBITRI.

El bosque mesófilo de montaña es el tipo de vegetación más importante por el número de especies endémicas y en riesgo de la REBITRI. Esto tiene sus implicaciones en la conservación de las especies listadas, principalmente porque aproximadamente $43 \%$ de las endémicas y en riesgo han sido reportados de este tipo de vegetación y $35 \%$ del total tienen como única referencia este ecosistema. Esto resalta la importancia de este ecosistema por su enorme riqueza de especies endémicas y en riesgo, y en general de una alta biodiversidad que ha sido reconocido para los bosques mesófilo montaña de México (Rzedowski, 1996; Luna-Vega et al., 2001; González-Espinosa et al., 2011).

La mayoría de las especies listadas en este trabajo han sido reportadas de vegetación primaria, y son pocas las especies encontradas en vegetación secundaria. Aunque no se indagaron las preferencias ecológicas de cada una de las especies, es posible indicar que la mayoría de éstas requieren condiciones ambientales óptimas que sólo los bosques conservados proporcionan. Por ejemplo, especies de las familias como Arecaceae, Bromeliaceae, Cyatheaceae, Lauraceae y Orchidaceae son muy estrictas en sus preferencias por hábitats en buen estado de conservación, no estableciéndose o siendo poco tolerante a zonas abiertas o con disturbio (Hodel,
1992; Lorea-Hernández, 2002; Espejo-Serna et al., 2005).

Los principales factores que afectan la conservación de las especies endémicas y en riesgo de la REBITRI son: cambio climático, cambio de uso de suelo, deforestación, extracción ilegal y sobrexplotación de productos forestales maderables y no maderables, fragmentación, incendios forestales y la reconversión de sistemas de producción de café de sombra (orgánico) a sistemas intensivos de producción (SEMARNAP, 1998; Schroth et al., 2009; Navarrete et al., 2010). El bosque mesófilo de montaña es uno de los ecosistemas más afectados (Toledo-Aceves et al., 2011). En la Sierra Madre de Chiapas este ecosistema cubría $16 \%$ de la región en la década de los setenta, para el año 2000 únicamente 6\%; la mayor parte de cobertura forestal perdida se encontraba en la REBITRI (Navarrete et al., 2010).

En este trabajo se listan 509 especies de plantas endémicas y en riesgo, lo que equivale al $17 \%$ de las especies que se distribuyen actualmente para la REBITRI. El número de especies es importante y resalta el valor efectivo de la reserva como área natural protegida. Sin embargo, es necesario incrementar los estudios florísticos y taxonómicos para tener más certidumbre en la validación y distribución de las especies presentes para la REBITRI, lo cual permitiría la inclusión o exclusión de especies. Por otra parte, nosotros sólo mencionamos las principales amenazas o factores que inciden sobre la conservación de las especies listadas, pero es necesario evaluar el riesgo real que éstos tienen sobre la conservación de las especies. No se cuenta con información sobre la ecología, biología y conservación de las especies consideradas en este trabajo, o al menos de las que se encuentren en los estatus más elevados de riesgo (p. ej., en peligro de extinción) o tengan una distribución geográfica muy limitada. Por lo que esperamos que con este trabajo se incentiven los estudios respectivos.

\section{Agradecimientos}

Este trabajo fue financiado por Christopher Davidson y Sharon Christoph a través del proyecto IFT01/05. Los autores agradecen a Angelita López Cruz, Jorge Martínez Meléndez, Manuel Martínez Meléndez, Héctor Gómez Domínguez y Mario Robles Molina por la ayuda en la conformación de la base de datos, a Andrew P. Vovides por la traducción al inglés del resumen, al apoyo otorgado por los curadores y personal de los herbarios CHIP, ECOSUR, INECOL, MEXU y MO, a Mario González Espinosa quien nos apoyó cediendo una versión preliminar de la obra "The Red List of Mexican Cloud Forest Trees".

\section{Literatura citada}

Acosta-Castellanos S. 2002. Plantas vasculares raras, amenazadas, o en peligro de extinción del Estado de Oaxaca, un panorama preliminar. Polibotánica 13:47-82. 
Borhidi A., Darók J., Kocsis M. y Stranczinger Sz. 2004. Critical revision of the Omiltemia Complex (Rubiaceae, Hamelieae). Acta Botanica Hungarica 46:69-76.

Borhidi A. 2006. Rubiáceas de México. Akadémiai Kiadó, Budapest.

Breedlove D. 1981. Introduction to Flora of Chiapas. Part 1. California Academy of Science, San Francisco.

Castillo-Campos G., Medina-Abreo M.E., Dávila-Aranda P.D. y Zavala-Hurtado J.A. 2005. Contribución al conocimiento del endemismo de la flora vascular en Veracruz, México. Acta Botanica Mexicana 73:19-97.

Challenger A. 1998. Utilización y Conservación de los Ecosistemas Terrestres de México. Pasado, Presente y Futuro. Comisión Nacional para el Conocimiento y Uso de la Biodiversidad/ Universidad Nacional Autónoma de México/Agrupación Sierra Madre S.C., México, D.F.

Ceballos G., Díaz-Pardo E., Espinosa H., Flores-Villela O., García A., Martínez L., Martínez-Meyer E., Navarro A., Ochoa L., Salazar I. y Santos-Barrera G. 2009. Zonas críticas y de alto riesgo para la conservación de la biodiversidad en México. En: Dirzo R., González R. y March I.J. Comps. Capital Natural de México, volumen II, Estado de Conservación y Tendencias de Cambio, pp. 575-600, Comisión Nacional para el Conocimiento y Uso de la Biodiversidad, México, D.F.

Contreras-Medina R. y Luna-Vega I. 2007. Species richness, endemism and conservation of Mexican Gymnosperms. Biodiversity and Conservation 16:1803-1821.

Crabbe J.A., Jermy A.C. y Mickel J.T. 1975. A new generis sequence for the Pteridophyte herbarium. Fern Gazette 11:141-162.

Cronquist A. 1988. The Evolution and Classification of the Flowering Plants. The New York Botanical Garden Press, Nueva York.

Dávila-Aranda P., Lira-Saade R. y Valdés-Reyna J. 2004. Endemic species of grasses in Mexico: a phytogeographic approach. Biodiversity and Conservation 13:1101-1121.

Davidse G., Sousa M.S. y Charter A.O. Eds. 1994. Flora Mesoamericana, Volumen 6: Alismataceae a Cyperaceae. Universidad Nacional Autónoma de México/Missouri Botanical Garden/The Natural History Museum, México, D.F.

Davidse G., Sousa M.S. y Knapp S. Eds. 1995. Flora Mesoamericana, Volumen 1: Psilotaceae a Salviniaceae. Universidad Nacional Autónoma de México/Missouri Botanical Garden/The Natural History Museum, México, D.F.

Davidse G., Sousa M.S., Knapp S. y Chiang F. Eds. 2009. Flora Mesoamericana, Volumen 4, parte 1: Cucurbitaceae a Polemoniaceae. Universidad Nacional Autónoma de México/Missouri Botanical Garden/The Natural History Museum, México, D.F.

Dirzo R. y Raven P.H. 2003. Global state of biodiversity and loss. Annual Review of Environment and Resources 28:137-167.

Espejo-Serna A., López-Ferrari A.R., Ramírez-Morillo I., Holst B.K., Luther H.E. y Till W. 2004. Checklist of Mexican Bromeliaceae with notes on species distribution and levels of endemism. Selbyana 25:33-86.

Espejo-Serna A., López-Ferrari A.R., Jiménez-Machorro R. y Sánchez-Saldaña L. 2005. Las orquídeas de los cafetales en México: una opción para el uso sostenible de ecosistemas tropicales. Revista de Biología Tropical 53:73-84.

Espinosa-Medinilla E., Anzures-Dadda A. y Cruz-Aldan E. 1998. Mamíferos de la reserva de la biosfera El Triunfo, Chiapas. Revista Mexicana de Mastozoología 3:79-94.
García-Cruz C.J. y Sosa V. 2011. Las orquídeas. En: Cruz-Angón A. Ed. La Biodiversidad en Veracruz, Estudio de Caso. Vol. II, pp. 191-199, Comisión Nacional para el Conocimiento y Uso de la Biodiversidad/Gobierno del Estado de Veracruz/Universidad Veracruzana/Instituto de Ecología, A.C., México, D.F.

García-Mendoza A., Tenorio-Lezama P. y Reyes-Santiago J. 1994. El endemismo en la flora fanerogámica de la mixteca alta de Oaxaca-Puebla, México. Acta Botanica Mexicana 27:52-73.

González-Espinosa M., Meave J.A., Lorea-Hernández F.G., Ibarra-Manríquez G. y Newton A.C. Eds. 2011. The Red List of Mexican Cloud Forest Trees. Fauna \& Flora International, Cambridge.

Graham A. 1998. Factores históricos de la diversidad biológica de México. En Ramamoorthy T.P., Bye R., Lot A. y Fa J. Comps. Diversidad Biológica de México, Orígenes y Distribución, pp. 109-127, Universidad Nacional Autónoma de México, México, D.F.

Hernández H.M. y Godínez-A. H. 1994. Contribución al conocimiento de las cactáceas mexicanas amenazadas. Acta Botanica Mexicana 26:33-52.

Hodel D.R. 1992. Chamaedorea Palms. The Species and their Cultivation. Allen Press, Lawrence.

IUCN [International Union for Conservation of Nature]. 2011. IUCN Red List of Threatened Species. Versión 2011.1. <http:// www.iucnredlist.org>. Revisado: Julio, 2011.

Knapp S. y Davidse G. 2006. Flora of Guatemala revisited. En: Cano E.B. Ed. Biodiversidad de Guatemala, Volumen I, pp. 2547, Universidad del Valle de Guatemala. Guatemala.

Long A. y Heath M. 1991. Flora of the El Triunfo Biosphere Reserve, Chiapas, Mexico. A preliminary floristic inventory and the plant communities of polygon I. Anales del Instituto de Biología, Serie Botánica 62:133-172.

Lorea-Hernández F.G. 2002. La familia Lauraceae en el sur de México: diversidad, distribución y estado de conservación. Boletín de la Sociedad Botánica de México 71:59-70.

Luna-Vega I., Morrone J.J., Alcántara-Ayala O. y Espinosa-Organista D. 2001. Biogeographical affinities among Neotropical cloud forest. Plant Systematics and Evolution 228:229-239.

Luna-Vega I., Alcántara-Ayala O. y Contreras-Medina R. 2004. Patterns of diversity, endemism and conservation: an example with Mexican species of Ternstroemiaceae Mirb. ex DC. (Tricolpates: Ericales). Biodiversity and Conservation 13:27232739.

Mabberley D.J. 1987. The Plant-Book. A Portable Dictionary of the Higher Plants. Cambridge University Press, Nueva York.

Martínez-Camilo R., Martínez-Meléndez N., Pérez-Farrera M.A. y Lorence D.H. 2011. Una nueva especie de Pseudomiltemia (Rubiaceae) de Chiapas, México. Brittonia 63:197-202.

Martínez-Meléndez J., Pérez-Farrera M.A. y Farrera-Sarmiento O. 2008. Inventario florístico del cerro El Cebú y zonas adyacentes en la Reserva de la Biosfera El Triunfo (Polígono V), Chiapas, México. Boletín de la Sociedad Botánica de México 82:21-40.

Martínez-Meléndez N., Pérez-Farrera M.A. y Martínez-Camilo R. 2009. The vascular epiphyte flora of the El Triunfo Biosphere Reserve, Chiapas, México. Rhodora 111:503-535.

Matuda E. 1950. A contribution to out knowledge of wild flora of Mt. Ovando. American Midland Naturalist 44:195-223.

Méndez-Larios L., Ortiz E. y Villaseñor J.L. 2004. Las Magnoliophyta endémicas de la porción xerofítica de la provincia florística del valle de Tehuacán-Cuicatlán, México. Anales del 
Instituto de Biología: Serie Botánica 75:87-104.

Mickel J. y Smith A.R. 2004. The Pteridophytes of Mexico. New York Botanical Garden Press, Nueva York.

Moreno N.P. 1984. Glosario Botánico Ilustrado. Instituto Nacional de Investigaciones sobre Recursos Bióticos, Xalapa.

Müllerried F.K.G. 1957. Geología de Chiapas. Gobierno del estado de Chiapas, Tuxtla Gutiérrez.

Navarrete D., Méndez D., Flamenco A. y Alba P. 2010. Situación actual, fragmentación, áreas prioritarias de conservación y principales amenazas del bosque mesófilo de Chiapas. En: Pérez-Farrera M.A., Tejeda-Cruz C. y Silva-Rivera E. Eds. Los Bosques Mesófilos de Montaña en Chiapas: Situación Actual, Diversidad y Conservación, pp. 295-326, Universidad de Ciencias y Artes de Chiapas, Tuxtla Gutiérrez.

Pérez-Farrera M.A. 2004. Flora y vegetación de la reserva de la biósfera El Triunfo: diversidad, riqueza y endemismo. En: Pérez-Farrera M.A., Martínez-Meléndez N., Hernández-Yáñez A. y Arreola-Muñoz A.V. Eds. La Reserva de la Biósfera El Triunfo, Tras una Década de Conservación, pp. 77-100, Universidad de Ciencias y Artes de Chiapas, Tuxtla Gutiérrez.

Pérez-Farrera M.A., Martínez-Camilo R., Martínez-Meléndez N., Farrera-Sarmiento O. y Maza-Villalobos S. 2012. Listado florístico del Cerro Quetzal (Polígono III) de la Reserva de la Biosfera El Triunfo, Chiapas, México. Botanical Sciences 90:113-142.

Rzedowski J. 1978. Vegetación de México. Limusa, México, D.F.

Rzedowski J. 1991a. Diversidad y orígenes de la flora fanerogámica de México. Acta Botanica Mexicana 14:3-21.

Rzedowski J. 1991b. El endemismo en la flora fanerogámica Mexicana: una apreciación analítica preliminar. Acta Botanica Mexicana 15:47-64.

Rzedowski J. 1996. Análisis preliminar de la flora vascular de los bosques mesófilos de montaña de México. Acta Botanica Mexicana 35:25-44.

Rzedowski J. 1998. Diversidad y orígenes de la flora fanerogámica de México. En: Ramamoorthy T.P., Bye R., Lot A. y Fa J. Eds. Diversidad Biológica de México, Orígenes y Distribución, pp. 129-145, Universidad Nacional Autónoma de México, México, D.F.

Rzedowski J. y Calderón-de Rzedowski G. 1989. Transisthmic Mexico (Campeche, Chiapas, Quintana Roo, Tabasco and Yucatán). En: Cambell D.G. y Hammond H.D. Eds. Floristic Inventory of Tropical Countries: The Plant Systematics, Collections, and Vegetation, plus Recommendations for the Future, pp. 270-280, The New York Botanical Garden, Nueva York.

Schroth G., Laderach P., Dempewolf J., Philpott S., Haggar J., Eakin H., Castillejos T., García-Moreno J., Soto-Pinto L., Hernández R., Eitzinger A. y Ramírez-Villegas J. 2009. Towards a climate change adaptation strategy for coffee communities and ecosystems in the Sierra Madre de Chiapas, Mexico. Mitigation and Adaptation Strategies for Global Change 14:605-625.

SEMARNAP [Secretaría del Medio Ambiente, Recursos Naturales y Pesca]. 1998. Programa de manejo de la Reserva de la Biosfera El Triunfo. Secretaria de Medio Ambiente, Recursos Naturales y Pesca. México, D.F.

SEMARNAT [Secretaría del Medio Ambiente y Recursos Naturales]. 2002. Norma Oficial Mexicana NOM-059-ECOL-2001, Protección ambiental-Especies nativas de México de flora y fauna silvestres-Categorías de riesgo y especificaciones para su inclusión, exclusión o cambio-Lista de especies en riesgo. Diario Oficial de la Federación. 2a Sección, 6 de marzo de 2002.

SEMARNAT [Secretaría del Medio Ambiente y Recursos Naturales]. 2010. Norma Oficial Mexicana NOM-059-SEMARNAT2010 Protección ambiental-Especies nativas de México de flora y fauna silvestres-Categorías de riesgo y especificaciones para su inclusión, exclusión o cambio-Lista de especies en riesgo. Diario Oficial de la Federación, 2a sección, 30 de diciembre de 2010.

Soto-Arenas M.A., Solano-Gómez R. y Hágsater E. 2007. Risk of extinction and patterns of diversity loss in Mexican orchids. Lankesteriana 7:114-121.

Toledo V.M. 1982. Pleistocene changes of vegetation in tropical Mexico. En: Prace G.T. Ed. Biological Diversification in the Tropics: Proceedings of the Fifth International Symposium of the Association for Tropical Biology, pp. 93-111, Columbia University Press, Nueva York.

Toledo-Aceves T., Meave J.A., González-Espinosa M. y Ramírez-Marcial N. 2011. Tropical montane cloud forests: current threats and opportunities for their conservation and sustainable management in Mexico. Journal of Environmental Management 92:974-981.

Turner B.L. 1997. The Comps of Mexico, a systematic account of the family Asteraceae, vol 1, Eupatorieae. Phytologia Memoirs 11:1-272.

Villaseñor J.L. 1991. Las Heliantheae endémicas a México: una guía hacia la conservación. Acta Botanica Mexicana 15:29-46.

Villaseñor J.L. 2003. Diversidad y distribución de las Magnoliophyta de México. Interciencia 28:160-167.

Villaseñor J.L. 2010. El Bosque Húmedo de Montaña en México y sus Plantas Vasculares: Catálogo Florístico-Taxonómico. Universidad Nacional Autónoma de México/Comisión Nacional para el Conocimiento y Uso de la Biodiversidad, México, D.F.

Vovides A.P., Luna V. y Medina G. 1997. Relación de algunas plantas y hongos mexicanos raros, amenazados o en peligro de extinción y sugerencias para su conservación. Acta Botanica Mexicana 39:1-42.

Recibido: 11 de noviembre de 2011

Aceptado: 27 de marzo de 2012 
Apéndice 1. Relación taxonómica de las especies de plantas endémicas y en riesgo presentes en la Reserva de la Biosfera El Triunfo, Chiapas. Estado de conservación según la Norma Oficial Mexicana (NOM; NOM-059-SEMARNAT-2010): A = amenazada, P = en peligro de extinción, $\mathrm{PR}=$ protección especial, y de la International Union for Conservation of Nature (IUCN): CR = en peligro crítico, $\mathrm{EN}=$ En peligro, $\mathrm{NT}=$ Casi amenazado, $\mathrm{VU}=$ Vulnerable. Hábito $(\mathrm{HA})$ : $\mathrm{Ab}=$ árbol, $\mathrm{Ar}=\mathrm{arbusto}, \mathrm{Ac}=$ arborescente, $\mathrm{H}=$ hierba terrestre, $\mathrm{He}=$ hierba epífita, $\mathrm{B}=$ bejuco. Tipo de vegetación (TV) (Rzedowski, 1978): bc = bosque de coníferas, bq = bosque de Quercus, bmm = bosque mesófilo de montaña, btc = bosque tropical caducifólio, btp = bosque tropical perenifólio, bts = bosque tropical subcaducifolio, va = vegetación acuática y subacuática, vs = vegetación secundaria. Distribución (DI): Tr = Reserva de la Biosfera El Triunfo, Ch = Chiapas, G = Guatemala, B = Belice, O = Oaxaca, V = Veracruz, Gu = Guerrero, S = El Salvador, $\mathrm{H}=$ Honduras, $\mathrm{CR}=$ Costa Rica, $\mathrm{Ne}=$ no endémica.

\begin{tabular}{llllllll}
\hline Grupo/Familia/Especie & NOM & IUCN & HA & TV & DI \\
\hline Pteridophyta
\end{tabular}

\section{Aspleniaceae}

Asplenium breedlovei A.R.Sm.

Asplenium dentatum L.

Asplenium solmsii Baker ex Hemsl.

Asplenium tuerckheimii Maxon

\section{Cyatheaceae}

Alsophila firma (Baker) D.S.Conant PR

Alsophila salvinii Hook.

Cyathea costaricensis (Mett. ex Kuhn) Domin

Cyathea divergens var. tuerckheimii (Maxon) R.M.Tryon

Cyathea fulva (M. Martens \& Galeotti) Fée

Nephelea mexicana (Schltdl. \& Cham.) R.M.Tryon

Sphaeropteris horrida (Liebm.) R.M.Tryon

\section{Dicksoniaceae}

Dicksonia sellowiana Hook.

\section{Dryopteridaceae}

Elaphoglossum albomarginatum A.R.Sm.

Nephrolepis cordifolia (L.) C.Presl

\section{Marattiaceae}

Marattia weinmanniifolia Liebm.

\section{Polypodiaceae}

Campyloneurum phyllitidis (L.) C.Presl.

Polypodium alavae A.R.Sm.

Serpocaulon triseriale (Sw.) A.R.Sm.

\section{Selaginellaceae}

Selaginella porphyrospora A.Braun

Selaginella pulcherrima Liebm. ex E.Fourn.

Selaginella steyermarkii Alston

\section{Coniferophyta}

\section{Cupressaceae}

Cupressus lusitanica Mill.
PR

P

A

$$
P
$$

PR

PR

$P$

PR

PR

PR

A

A

P

PR

$\begin{array}{lll}\mathrm{H} & \text { bc, bmm } & \mathrm{Ch}, \mathrm{G} \\ \mathrm{H} & \text { bmm } & \mathrm{Ne} \\ \mathrm{H} & \text { bmm, btp } & \mathrm{Ch}, \mathrm{G} \\ \mathrm{H} & \text { bmm, btp } & \mathrm{Ch}, \mathrm{G}\end{array}$

AC

bmm

$\mathrm{Ne}$

bmm

bmm

$\mathrm{Ne}$

Ac

bmm

$\mathrm{Ne}$

AC

bmm, btp

$\mathrm{Ne}$

Ac

Ac

bmm, btp

$\mathrm{Ne}$

bc, bq, bmm

$\mathrm{Ne}$

Ac

Ac

bmm

$\mathrm{Ne}$

$\mathrm{He}$

bmm

$\mathrm{Ch}$

He bmm

$\mathrm{Ne}$

Ac

bmm

$\mathrm{Ne}$

bmm

$\mathrm{Ne}$

bmm

$\mathrm{Ch}$

$\mathrm{He}$

$\mathrm{He}$

bq, bmm, btp,

$\mathrm{Ne}$

bts, vs

$\begin{array}{lll}\mathrm{H} & \text { bmm, btp } & \mathrm{Ne} \\ \mathrm{H} & \text { btp } & \text { Ch, V } \\ \mathrm{H} & \text { bmm } & \text { Ch, G }\end{array}$

$\mathrm{Ab} \quad \mathrm{bc}, \mathrm{bmm}$

$\mathrm{Ne}$ 
Apéndice 1. Continuación

\begin{tabular}{|c|c|c|c|c|c|}
\hline Grupo/Familia/Especie & NOM & IUCN & HA & TV & DI \\
\hline \multicolumn{6}{|l|}{ Pinaceae } \\
\hline Abies guatemalensis Rehder & $\mathrm{P}$ & $\mathrm{VU}$ & $\mathrm{Ab}$ & bc & $\mathrm{Ne}$ \\
\hline Pinus ayacahuite C.Ehrenb. ex Schltdl. & & $\mathrm{VU}$ & $\mathrm{Ab}$ & bc & $\mathrm{Ne}$ \\
\hline \multicolumn{6}{|l|}{ Podocarpaceae } \\
\hline Podocarpus matudae Lundell & PR & EN & $A b$ & bc, bmm, btp, bts & $\mathrm{Ne}$ \\
\hline \multicolumn{6}{|l|}{ Cycadophyta } \\
\hline \multicolumn{6}{|l|}{ Zamiaceae } \\
\hline Ceratozamia matudae Lundell & $\mathrm{P}$ & EN & $\mathrm{H}$ & bc, bmm, btp & $\mathrm{Ch}, \mathrm{G}$ \\
\hline Ceratozamia vovidesii Pérez Farrera \& Iglesias & $\mathrm{P}$ & $\mathrm{VU}$ & Ac & bc, bq, bmm, btp & $\mathrm{Ch}$ \\
\hline Zamia herrerae Calderón \& Standl. & PR & VU & Ac & btp & $\mathrm{Ch}, \mathrm{G}, \mathrm{N}, \mathrm{S}$ \\
\hline Zamia soconuscensis Schutzman, Vovides \& Dehgan & $\mathrm{P}$ & VU & $\mathrm{H}$ & btp, bts & $\operatorname{Tr}$ \\
\hline
\end{tabular}

\section{Liliopsida}

Agavaceae

Beschorneria albiflora Matuda

bmm

Ch, O, V

\section{Araceae}

Anthurium andicola Liebm.

Anthurium cerrobaulense Matuda

Anthurium chamulense Matuda subsp. chamulense

Anthurium chiapasense Standl. subsp. chiapasense

Anthurium nakamurae Matuda

Anthurium ovandense Matuda

Anthurium rzedowskii Croat

Anthurium sarukhanianum Croat \& Haager

Anthurium schlechtendalii subsp. jimenezii (Matuda) Croat

Anthurium seleri Engl.

Anthurium titanium Standl. \& Steyerm.

Dieffenbachia oerstedii Schott

Philodendron escuintlense Matuda

Philodendron radiatum var. pseudoradiatum (Matuda) Croat

Philodendron seguine Schott subsp. seguine

Syngonium steyermarkii Croat

$\begin{array}{lll}\text { H } & \text { bmm } & \text { Ch, O, V } \\ \text { He } & \text { bmm, btp, bts } & \text { Ch, O } \\ \text { He } & \text { bmm } & \text { Ch } \\ \text { He } & \text { bmm, btp, bts, vs } & \text { Ch, G } \\ \text { He } & \text { bmm, btp } & \text { Ch } \\ \text { He } & \text { bmm } & \text { Ch, O } \\ \text { He } & \text { bmm, vs } & \text { Ch, O, V } \\ \text { He } & \text { bmm, btp, bts } & \text { Ch, Gu } \\ \text { He } & \text { bts, btc } & \text { Ch, O, V } \\ \text { H } & \text { btp } & \text { Ch, G } \\ \text { H } & \text { bc, bmm, btp } & \text { Ch, G } \\ \text { H } & \text { btp } & \text { Ne } \\ \text { B } & \text { bmm } & \text { Ch, O, V } \\ \text { B } & \text { btp } & \text { Ch } \\ \text { B } & \text { bmm } & \text { Ch, O } \\ \text { B } & \text { bmm } & \text { Ch, G }\end{array}$

\section{Arecaceae}

Chamaedorea fractiflexa Hodel \& J.J.Castillo

Chamaedorea graminifolia $\mathrm{H}$. Wendl.

Chamaedorea ibarrae Hodel

Chamaedorea keeleriorum Hodel \& J.J.Castillo

Chamaedorea nubium Standl. \& Steyerm.

Chamaedorea pinnatifrons (Jacq.) Oerst.

Chamaedorea quezalteca Standl. \& Steyerm.

Chamaedorea woodsoniana L.H.Bailey

Cryosophila nana (Kunth) Blume ex Salomon

Geonoma pinnatifrons subsp. membranacea

(H.Wendl. ex Spruce) Aj.Mend

Geonoma undulata Klotzsch.
A

A

$\begin{array}{ll}\mathrm{A} & \mathrm{AC} \\ \mathrm{A} & \mathrm{AC}\end{array}$

A $\quad A C$

A $A C$

A

NT

Ac

Ac

Ac

Ac

Ac

Ac

Ac

AC

bmm, btp

bmm, btp, bts

bc, bmm, vs

bc, bmm, btp

bq, bmm, btp

$\mathrm{Ne}$

Ch, G, V

Ch, G

Ch, G

bts, vs

bmm, btp, bts

bmm, btp

btc, btp

btp

Ac

Ac

A

bmm, btp

$\mathrm{Ne}$
$\mathrm{Ne}$

$\mathrm{Ne}$

$\mathrm{Ne}$

Ch, G, H, S

$\mathrm{Ne}$

Ch, G 
Apéndice 1. Continuación

\begin{tabular}{|c|c|c|c|c|c|}
\hline Grupo/Familia/Especie & NOM & IUCN & HA & TV & DI \\
\hline \multicolumn{6}{|l|}{ Bromeliaceae } \\
\hline Aechmea matudae L.B.Sm. & & & $\mathrm{He}$ & btp & $\mathrm{Ch}$ \\
\hline Catopsis berteroniana (Schult. \& Schult.f.) Mez & PR & & $\mathrm{He}$ & bc & $\mathrm{Ne}$ \\
\hline Pitcairnia breedlovei L.B.Sm. & & & $\mathrm{H}$ & btp & $\mathrm{Ch}$ \\
\hline Pitcairnia calderonii Standl. \& L.B.Sm. & & & $\mathrm{H}$ & bmm & $\mathrm{Ch}, \mathrm{B}$ \\
\hline Pitcairnia matudae L.B.Sm. & & & $\mathrm{H}$ & bmm & $\operatorname{Tr}$ \\
\hline Pitcairnia saxicola L.B.Sm. & & & $\mathrm{He}$ & bmm & $\mathrm{Ch}, \mathrm{H}, \mathrm{CR}$ \\
\hline Pitcairnia tuerckheimii Donn.Sm. & & & $\mathrm{H}$ & btp & Ch, G \\
\hline Tillandsia concolor L.B.Sm. & A & & $\mathrm{He}$ & bc, btp, bts & $\mathrm{Ne}$ \\
\hline Tillandsia eizii L.B.Sm. & & & $\mathrm{He}$ & bc & Ch, G \\
\hline Tillandsia fuchsii W.Till & & & $\mathrm{He}$ & btp, bts, vs & $\mathrm{Ch}, \mathrm{O}$ \\
\hline Tillandsia heterophylla E.Morren & & & $\mathrm{He}$ & bmm, btp & Ch, V \\
\hline Tillandsia imperialis E.Morren ex Mez & A & & $\mathrm{He}$ & bmm & $\mathrm{Ne}$ \\
\hline Tillandsia lampropoda L.B.Sm. & A & & $\mathrm{He}$ & bc, bmm & $\mathrm{Ne}$ \\
\hline Tillandsia lautneri Ehlers & & & $\mathrm{He}$ & bc, bq, bmm & Ch, G \\
\hline Tillandsia ponderosa L.B.Sm. & $A$ & & $\mathrm{He}$ & bmm, btp, bts & $\mathrm{Ne}$ \\
\hline Tillandsia seleriana Mez & A & & $\mathrm{He}$ & bc, bq & $\mathrm{Ne}$ \\
\hline Tillandsia tricolor Schltdl. \& Cham. & A & & $\mathrm{He}$ & bmm & $\mathrm{Ne}$ \\
\hline Tillandsia vriesioides Matuda & & & $\mathrm{He}$ & bmm & $\mathrm{Ch}$ \\
\hline Vriesea pectinata L.B.Sm. & & & $\mathrm{He}$ & btp & Ch, G \\
\hline Vriesea pycnantha L.B.Sm. = V. ovandensis Matuda & A & & $\mathrm{He}$ & bmm & Ch, G \\
\hline Vriesea werckleana Mez = V. breedloveana L.B.Sm. & A & & $\mathrm{He}$ & bmm & $\mathrm{Ne}$ \\
\hline Werauhia nocturna (Matuda) J.R.Grant & & & $\mathrm{He}$ & bmm & $\mathrm{Ch}, \mathrm{O}$ \\
\hline \multicolumn{6}{|l|}{ Commelinaceae } \\
\hline Tradescantia deficiens Brandegee & & & $\mathrm{H}$ & bc, bmm & Ch, G \\
\hline Tradescantia plusiantha Standl. & & & $\mathrm{H}$ & bmm, bts & $\mathrm{Ch}, \mathrm{O}$ \\
\hline Tradescantia zebrina var. mollipila D.Hunt & & & $\mathrm{H}$ & bc, bmm & $\mathrm{Ch}$ \\
\hline \multicolumn{6}{|l|}{ Cyperaceae } \\
\hline Cyperus matudae G.Tucker & & & $\mathrm{H}$ & $\mathrm{bq}$ & $\mathrm{Ch}$ \\
\hline \multicolumn{6}{|l|}{ Dioscoreaceae } \\
\hline Dioscorea carionis Prain \& Burkill & & & $\mathrm{B}$ & btp & $\mathrm{Ch}, \mathrm{G}$ \\
\hline Dioscorea gomez-pompae O.Téllez & & & B & bc & $\mathrm{Ch}$ \\
\hline Dioscorea remota C.V.Morton & & & $\mathrm{B}$ & bts & $\mathrm{Ch}, \mathrm{CR}, \mathrm{N}, \mathrm{P}$ \\
\hline Dioscorea spiculiflora var. chiapasana Gómez-Pompa & & & $\mathrm{B}$ & bc & $\mathrm{Ch}$ \\
\hline Dioscorea sumiderensis B.G.Schubert \& O.Téllez & & & B & bts & $\mathrm{Ch}$ \\
\hline \multicolumn{6}{|l|}{ Liliaceae } \\
\hline Maianthemum flexuosum (Bertol) LaFrankie & & & $\mathrm{H}$ & bc, bmm, btp & Ch, G \\
\hline \multicolumn{6}{|l|}{ Orchidaceae } \\
\hline Acianthera breedlovei Soto Arenas, Solano \& Salazar & & & $\mathrm{He}$ & bc & $\mathrm{Ch}$ \\
\hline Cranichis cochleata Dressler & & & $\mathrm{H}$ & bmm & $\mathrm{Ch}, \mathrm{G}, \mathrm{V}$ \\
\hline Cypripedium irapeanum La Llave \& Lex. & A & & $\mathrm{He}$ & bc & $\mathrm{Ne}$ \\
\hline Epidendrum cerinum Schltr. & PR & & $\mathrm{He}$ & bmm & $\mathrm{Ne}$ \\
\hline Epidendrum erectifolium Hágsater \& L.SánchezS. & & & $\mathrm{He}$ & bts & $\mathrm{Ch}$ \\
\hline $\begin{array}{l}\text { Epidendrum tacanaense Hágsater, Soto Arenas } \\
\text { \& E.SantiagoA. }\end{array}$ & & & $\mathrm{He}$ & bmm & $\mathrm{Ch}$ \\
\hline
\end{tabular}


Apéndice 1. Continuación

\begin{tabular}{|c|c|c|c|c|c|}
\hline Grupo/Familia/Especie & NOM & IUCN & HA & TV & DI \\
\hline Erycina crista-galli (Rchb.f.) N.H.Williams \& M.W.Chase & PR & & $\mathrm{He}$ & bts & $\mathrm{Ne}$ \\
\hline Guarianthe skinneri (Bateman) Dressler \& W.E.Higgins & A & & $\mathrm{He}$ & $\begin{array}{l}\text { bq, bmm, btc, } \\
\text { btp, bts }\end{array}$ & $\mathrm{Ne}$ \\
\hline Habenaria tetranema Schltr. & & & $\mathrm{H}$ & bmm & $\mathrm{Ch}, \mathrm{G}, \mathrm{S}$ \\
\hline Lepanthes matudana Salazar \& Soto Arenas & & & $\mathrm{He}$ & bmm, btp, va & $\mathrm{Ch}, \mathrm{G}$ \\
\hline Lepanthes tenuiloba R.E.Schult. \& Dillon & & & $\mathrm{He}$ & btp & Ch, G \\
\hline Lockhartia verrucosa Lindl. ex Rchb. F. & & & $\mathrm{He}$ & bc, btp & $\mathrm{Ch}, \mathrm{O}$ \\
\hline Mormodes nagelii L.O.Williams & & & $\mathrm{He}$ & bmm, btp & Ch, G \\
\hline Mormodes tuxtlensis Salazar & & & $\mathrm{He}$ & bc & Ch, V \\
\hline Oncidium leucochilum Bateman ex Lindl. & A & & $\mathrm{He}$ & bmm & $\mathrm{Ch}, \mathrm{G}, \mathrm{H}$ \\
\hline Oncidium ochmatochilum Rchb.f. & A & & $\mathrm{He}$ & bmm & $\mathrm{Ne}$ \\
\hline Oncidium suttonii Bateman ex Lindl. & A & & $\mathrm{He}$ & btp & $\mathrm{Ch}, \mathrm{G}, \mathrm{S}$ \\
\hline Pleurothallis endotrachys Rchb.f. & PR & & $\mathrm{He}$ & bmm & $\mathrm{Ne}$ \\
\hline Pleurothallis saccatilabia C.Schweinf. & $\mathrm{PR}$ & & $\mathrm{He}$ & bmm & $\mathrm{Ch}, \mathrm{CR}, \mathrm{G}$ \\
\hline Prosthechea vitellina (Lindl.) W.E.Higgins & PR & & $\mathrm{He}$ & bc, bmm & $\mathrm{Ne}$ \\
\hline Rhynchostele cordata (Lindl.) Soto Arenas \& Salazar & A & & $\mathrm{He}$ & bmm, vs & $\mathrm{Ne}$ \\
\hline Rhynchostele rossii (Lindl.) Soto Arenas \& Salazar & A & & $\mathrm{He}$ & bmm & $\mathrm{Ne}$ \\
\hline Rossioglossum grande (Lindl.) Garay \& G.C.Kenn. & $\mathrm{P}$ & & $\mathrm{He}$ & bmm & $\mathrm{Ch}, \mathrm{B}$ \\
\hline Scelochilus tuerckheimii Schltr. & A & & $\mathrm{He}$ & bmm & $\mathrm{Ne}$ \\
\hline Trichosalpinx cedralensis (Ames) Luer & PR & & $\mathrm{He}$ & bmm & $\mathrm{Ne}$ \\
\hline \multicolumn{6}{|l|}{ Poaceae } \\
\hline Chusquea repens L.G.Clark \& Londoño subsp. repens & & & $\mathrm{H}$ & bc & $\mathrm{Ch}, \mathrm{O}$ \\
\hline Chusquea sulcata Swallen & & & $\mathrm{H}$ & bmm & $\mathrm{Ch}, \mathrm{CR}, \mathrm{G}, \mathrm{P}$ \\
\hline Echinolaena standleyi (A.Hicthc.) Stieber & & & $\mathrm{H}$ & bmm, bc & $\mathrm{Ch}, \mathrm{B}, \mathrm{G}, \mathrm{H}$ \\
\hline Muhlenbergia breviligula A.Hitchc. in Britton & & & $\mathrm{H}$ & bc & $\mathrm{Ch}, \mathrm{G}, \mathrm{H}, \mathrm{N}$ \\
\hline Muhlenbergia xanthodas Soderstrom & & & $\mathrm{H}$ & bmm & $\operatorname{Tr}$ \\
\hline \multicolumn{6}{|l|}{ Smilacaceae } \\
\hline Smilax chiapensis Lundell & & & B & btp & $\mathrm{Ch}$ \\
\hline Smilax purpusii Brandegee & & & B & btp & $\mathrm{Ch}, \mathrm{O}$ \\
\hline \multicolumn{6}{|l|}{ Magnoliopsida } \\
\hline \multicolumn{6}{|l|}{ Acanthaceae } \\
\hline Justicia inaequalis Benth. & & & $\mathrm{Ar}$ & bmm & $\mathrm{Ch}, \mathrm{G}$ \\
\hline Justicia madrensis T.F.Daniel & & & $\operatorname{Ar}$ & btp & $\mathrm{Ch}$ \\
\hline Justicia mirandae T.F.Daniel & & & $\operatorname{Ar}$ & bc & $\mathrm{Ch}$ \\
\hline Ruellia matudae Leonard & & & $\mathrm{H}$ & bc, btp & $\mathrm{Ch}$ \\
\hline Stenostephanus chiapensis T.F.Daniel & & & $\mathrm{H}$ & bmm & $\mathrm{Ch}$ \\
\hline Stenostephanus glabrus (Leonard) T.F.Daniel & & & $\mathrm{H}$ & bmm, bts & Ch, G \\
\hline Stenostephanus monolophus (Donn.Sm.) T.F.Daniel & & & $\operatorname{Ar}$ & btp & $\mathrm{Ch}, \mathrm{G}$ \\
\hline Spathacanthus hahnianus Baill. & & VU & $\operatorname{Ar}$ & bmm & $\mathrm{Ne}$ \\
\hline Spathacanthus parviflorus Leonard & & $\mathrm{VU}$ & $\operatorname{Ar}$ & bmm, va & Ch, G \\
\hline \multicolumn{6}{|l|}{ Actinidiaceae } \\
\hline Saurauia aspera Turcz. & & $\mathrm{VU}$ & $\mathrm{Ab}$ & bc, bmm, btp & $\mathrm{Ne}$ \\
\hline Saurauia conzattii Buscal. & & EN & $\mathrm{Ab}$ & bmm & $\mathrm{Ch}, \mathrm{G}, \mathrm{H}, \mathrm{O}$ \\
\hline Saurauia kegeliana Schltdl. & & $\mathrm{VU}$ & $\mathrm{Ab}$ & bc, bq, bmm, btp & $\mathrm{Ch}, \mathrm{G}, \mathrm{H}, \mathrm{S}$ \\
\hline Saurauia madrensis B.T.Keller \& Breedlove & & $\mathrm{EN}$ & $\mathrm{Ab}$ & bc, bmm, btp & $\mathrm{Ch}$ \\
\hline Saurauia matudae Lundell & & EN & $\mathrm{Ab}$ & $\mathrm{bc}, \mathrm{bmm}$ & $\mathrm{Ch}, \mathrm{O}$ \\
\hline
\end{tabular}


Apéndice 1. Continuación

\begin{tabular}{|c|c|c|c|c|c|}
\hline Grupo/Familia/Especie & NOM & IUCN & HA & TV & DI \\
\hline Saurauia oreophila Hemsl. & & VU & $\mathrm{Ab}$ & bmm & $\mathrm{Ch}, \mathrm{G}, \mathrm{H}, \mathrm{O}$ \\
\hline Saurauia scabrida Hemsl. & & NT & $\mathrm{Ab}$ & bmm & $\mathrm{Ne}$ \\
\hline
\end{tabular}

\section{Anacardiaceae}

Astronium graveolens Jacq. Comocladia guatemalensis Donn.Sm.

Mauria sessiliflora Standl.

Tapirira mexicana Marchand

\section{Annonaceae}

Annona diversifolia Saff.

Annona scleroderma Saff.

Cymbopetalum stenophyllum Donn.Sm.

Desmopsis lanceolata Lundell

Sapranthus chiapensis G.E.Schatz

Rollinia membranaceae Triana \& Planch.

\section{Apiaceae}

Donnellsmithia serrata (J.M.Coult. \& Rose) Mathias

\& Constance

Myrrhidendron donnellsmithii J.M.Coult. \& Rose

Rhodosciadium nelsoni (J.M.Coult. \& Rose) Mathias

$\&$ Constance

\section{Apocynaceae}

Alstonia longifolia (A.DC.) Pichon

Prestonia speciosa Donn.Sm.

Thenardia galeottiana Baill.

Stemmadenia litoralis (Kunth) L.Allorge

\section{Aquifoliaceae}

Ilex belizensis Lundell

Ilex liebmannii Standl.

Ilex chiapensis Lundell

Ilex quercetorum I.M.Johnst.

Ilex tolucana Hemsl.

\section{Araliaceae}

Dendropanax pallidus M.J.Cannon \& Cannon

Dendropanax populifolius (Marchal) A.C.Sm.

Oreopanax arcanus A.C.Sm.

Oreopanax capitatus (Jacq.) Decne. \& Planch.

Oreopanax echinops (Cham. \& Schltdl.) Decne. \& Planch.

Oreopanax peltatus Linden

Oreopanax sanderianus Hemsl.

Oreopanax xalapensis (Kunth) Decne. \& Planch.
VU

A

VU

$\begin{array}{llll} & \text { Ab } & \text { btp } & \text { Ch, G, H, S } \\ & \text { Ab } & \text { btp } & \text { Ch, B, G, H } \\ & \text { Ab } & \text { btp, bts } & \text { Ch, G } \\ \text { CR } & \text { Ab } & \text { bmm, btp, bts } & \text { Ch } \\ & \text { H } & \text { btp } & \text { Tr } \\ \text { VU } & \text { Ar } & \text { btp } & \text { Ne }\end{array}$

$\mathrm{H} \quad$ bc, bmm

Ch, O

H bts

H btp

VU

VU

$$
\mathrm{Ab}
$$

$\mathrm{Ab}$

B

$\mathrm{Ab}$

$\mathrm{Ab}$

$\mathrm{Ab}$

$\mathrm{Ab}$

$\mathrm{Ab}$

$\mathrm{Ab}$

$\mathrm{Ab}$

bq

bmm

btp

bmm

bmm

Ch, CR, G, P

$\mathrm{Ch}$

$\mathrm{Ne}$

Ch, G, H, N

$\mathrm{Ch}, \mathrm{Gu}, \mathrm{O}$

$\mathrm{Ne}$

Ch, B

Ch, B

$\mathrm{Ch}, \mathrm{H}$

$\mathrm{Ne}$

$\mathrm{Ne}$

$\begin{array}{llll}\text { CR } & \text { Ab } & \text { bq, bmm, btp } & \text { Ch, G, S } \\ \text { CR } & \text { Ab } & \text { bmm } & \text { Ch, O } \\ \text { CR } & \text { Ab } & \text { bmm, btp } & \text { Ch, G } \\ \text { NT } & \text { Ab } & \text { bmm, btp } & \text { Ne } \\ \text { VU } & \text { Ab } & \text { bc, bmm, btp } & \mathrm{Ne} \\ \text { NT } & \text { Ab } & \text { bmm, btp } & \mathrm{Ne} \\ \text { EN } & \text { Ab } & \text { bmm, btp } & \text { Ne } \\ \text { NT } & \text { Ab } & \text { bmm, btp } & \text { Ne } \\ & & & \\ & & & \text { Ch, G } \\ & \text { B } & \text { btp } & \text { Ch, N, S } \\ & \text { B } & \text { btp } & \end{array}$

\section{Asclepiadaceae}

Gonolobus chiapensis (Brandegee) Woodson

Gonolobus cuajayote W.D.Stevens 
Apéndice 1. Continuación

\begin{tabular}{|c|c|c|c|c|c|}
\hline Grupo/Familia/Especie & NOM & IUCN & HA & TV & DI \\
\hline Gonolobus exannulatus W.D.Stevens & & & B & bmm & Ch, G \\
\hline Gonolobus stenosepalus (Donn.Sm.) Woodson & & & B & bc & $\mathrm{Ch}, \mathrm{G}, \mathrm{H}$ \\
\hline Marsdenia bourgaeana (Baill.) W.Rothe & & & B & bmm & $\mathrm{Ch}, \mathrm{G}, \mathrm{V}$ \\
\hline Marsdenia schlechteriana W.Rothe & & & B & bmm & $\mathrm{Ch}, \mathrm{G}, \mathrm{S}$ \\
\hline Marsdenia stephanotidifolia Woodson & & & B & btp & $\mathrm{Ch}, \mathrm{G}, \mathrm{S}$ \\
\hline Matelea inops Woodson & & & B & bc,bmm,btp & $\mathrm{Ch}, \mathrm{O}$ \\
\hline Orthosia cynanchoides W.D.Stevens & & & B & bmm & $\mathrm{Ch}$ \\
\hline Orthosia stipitata W.D.Stevens & & & B & bmm & $\operatorname{Tr}$ \\
\hline Pherotrichis villosa (Schult.) Meisn. & & & B & btp & $\mathrm{Ch}, \mathrm{G}, \mathrm{O}$ \\
\hline \multicolumn{6}{|l|}{ Asteraceae } \\
\hline Archibaccharis aequivenia (S.F.Blake) D.L.Nash & & & $\mathrm{Ar}$ & bmm & $\mathrm{Ch}, \mathrm{G}$ \\
\hline Archibaccharis androgyna (Brandegee) S.F.Blake & & & $\mathrm{Ar}$ & bmm & $\mathrm{Ch}, \mathrm{G}$ \\
\hline Bartlettina pinabetensis (B.L.Rob.) R.M.King \& H.Rob. & & & $\mathrm{Ar}$ & bmm & $\mathrm{Ch}, \mathrm{G}$ \\
\hline Calea megacephala B.L.Rob. \& Greenm. & & & $\mathrm{H}$ & btp & $\mathrm{Ch}, \mathrm{O}$ \\
\hline $\begin{array}{l}\text { Decachaeta ovandensis (Grashoff \& Beaman) } \\
\text { R.M.King \& H.Rob. }\end{array}$ & & & $\mathrm{H}$ & bmm & $\operatorname{Tr}$ \\
\hline Desmanthodium perfoliatum Benth. & & & $\mathrm{Ab}$ & btp & $\mathrm{Ch}, \mathrm{O}$ \\
\hline Eupatoriastrum angulifolium (B.L.Rob.) R.M.King \& H.Rob. & & & $\mathrm{H}$ & bmm & $\mathrm{Ch}, \mathrm{G}$ \\
\hline Eupatorium heathiae B.L.Turner & & & $\mathrm{Ar}$ & $\mathrm{bq}, \mathrm{bmm}$ & $\operatorname{Tr}$ \\
\hline Eupatorium heydeanum B.L.Rob. & & & $\mathrm{Ar}$ & btp & $\mathrm{Ch}, \mathrm{G}$ \\
\hline Eupatorium molinae L.O.Williams & & & $\mathrm{Ar}$ & bmm, btp & $\mathrm{Ch}, \mathrm{G}$ \\
\hline Eupatorium opadoclinium (S.F.Blake) McVaugh & & & $\mathrm{Ar}$ & btp & $\mathrm{Ch}$ \\
\hline Eupatorium siltepecanum B.L.Turner & & & $\mathrm{Ar}$ & bmm & $\operatorname{Tr}$ \\
\hline Eupatorium tenejapanum B.L.Turner & & & $\mathrm{H}$ & bmm & $\mathrm{Ch}, \mathrm{G}$ \\
\hline Gymnolaena chiapasana Strother & & & $\operatorname{Ar}$ & btp & $\mathrm{Ch}$ \\
\hline Koanophyllon pittieri (Klatt) R.M.King \& H.Rob. & & VU & $\mathrm{Ab}$ & bmm & $\mathrm{Ne}$ \\
\hline Montanoa pteropoda S.F.Blake & & & B & bmm & $\mathrm{Ch}, \mathrm{B}$ \\
\hline Montanoa standleyi V.A.Funk & & & $\mathrm{Ar}$ & bmm & $\mathrm{Ch}, \mathrm{O}$ \\
\hline Nesomia chiapensis B.L.Turner & & & $\mathrm{H}$ & btp & $\operatorname{Tr}$ \\
\hline Perymenium chloroleucum S.F.Blake & & & $\mathrm{Ar}$ & bts & $\mathrm{Ch}, \mathrm{G}, \mathrm{O}$ \\
\hline Perymenium gracile Hemsl. & & & $\mathrm{Ar}$ & bq & $\mathrm{Ch}, \mathrm{O}$ \\
\hline Perymenium klattianum J.J.Fay & & & $\mathrm{Ar}$ & bmm & $\mathrm{Ch}, \mathrm{V}$ \\
\hline Podachaenium chiapanum B.L.Turner & & & $\mathrm{H}$ & btp & $\mathrm{Ch}$ \\
\hline Roldana greenmanii H.Rob. \& Brettell & & & $\mathrm{Ar}$ & bmm & $\mathrm{Ch}, \mathrm{G}$ \\
\hline Rojasianthe superba Standl. \& Steyerm. & & & $\mathrm{Ar}$ & bmm & $\mathrm{Ch}, \mathrm{G}$ \\
\hline Salmea orthocephala Standl. \& Steyerm. & & & $\mathrm{H}$ & bts & $\mathrm{Ch}, \mathrm{B}, \mathrm{G}, \mathrm{H}$ \\
\hline Schistocarpha matudae H.Rob. & & & $\mathrm{H}$ & bmm & $\mathrm{Ch}$ \\
\hline Senecio cristobalensis Greenm. & & & $\mathrm{Ar}$ & bc & $\mathrm{Ch}, \mathrm{G}$ \\
\hline Stevia chiapensis Grashoff & & & $\mathrm{H}$ & bmm & $\mathrm{Ch}$ \\
\hline Stevia polycephala Bertol. & & & $\mathrm{Ar}$ & bc & $\mathrm{Ch}, \mathrm{O}$ \\
\hline Telanthophora cobanensis (J.M.Coult.) H.Rob. \& Brettell & & EN & $\operatorname{Ar}$ & bc, bmm, btp, vs & $\mathrm{Ne}$ \\
\hline Verbesina apleura S.F.Blake & & & $\mathrm{Ar}$ & btp & $\mathrm{Ch}, \mathrm{G}$ \\
\hline Wamalchitamia appressipila (S.F.Blake) Strother & & & $\mathrm{Ar}$ & bmm & $\mathrm{Ch}$ \\
\hline Wedelia purpurea (Greenm.) B.L.Turner & & & $\mathrm{Ar}$ & bc, bts & $\mathrm{Ch}, \mathrm{O}$ \\
\hline \multicolumn{6}{|l|}{ Begoniaceae } \\
\hline Begonia bettinae Ziesenh. & & & $\mathrm{H}$ & btp & $\mathrm{Ch}$ \\
\hline Begonia chiapensis Burt-Utley & & & $\mathrm{H}$ & btp & $\mathrm{Ch}$ \\
\hline Begonia corozoensis Ziesenh. & & & $\mathrm{H}$ & bmm & $\mathrm{Ch}$ \\
\hline
\end{tabular}


Apéndice 1. Continuación

\section{Grupo/Familia/Especie}

Begonia kenworthyae Ziesenh.

Begonia philodendroides Ziesenh.

\section{Berberidaceae}

Berberis chiapensis (Lundell) Lundell

\section{Betulaceae}

Carpinus caroliniana Walter

Ostrya virginiana (Mill.) K.Koch

\section{Bignoniaceae}

Amphitecna breedlovei A.H.Gentry

Amphitecna montana L.O.Williams

Amphitecna sessilifolia (Donn.Sm.) L.O.Williams

Amphitecna steyermarkii (A.H.Gentry) A.H.Gentry

Gibsoniothamnus cornutus (Donn.Sm.) A.H.Gentry

\section{Boraginaceae}

Varronia foliosa (M.Martens \& Galeotti) Borhidi

\section{Cactaceae}

Disocactus macdougallii (Alexander) Barthlott

Disocactus nelsonii (Britton \& Rose) Linding.

Weberocereus glaber (Eichlam) G.D.Rowley

\section{Cannabaceae}

Lozanella enantiophylla (Donn.Sm.) Killip \& C.V.Morton

\section{Capparaceae}

Forchhammeria matudae Lundell

Steriphoma clara Standl.

\section{Caprifoliaceae}

Viburnum acutifolium Benth.

Viburnum blandum C.V.Morton

Viburnum jucundum C.V.Morton

\section{Celastraceae}

Celastrus chiapensis Lundell

Celastrus siltepecanus Lundell

Maytenus chiapensis Lundell

Maytenus matudae Lundell

Maytenus stipitata Lundell

Microtropis contracta Lundell

Rhacoma riparia Lundell

Zinowiewia matudae Lundell

Zinowiewia rubra Lundell

\section{Chrysobalanaceae}

Couepia polyandra (Kunth) Rose

\begin{tabular}{|c|c|c|c|c|}
\hline NOM & IUCN & HA & TV & DI \\
\hline & & $\mathrm{H}$ & btp & $\mathrm{Ch}$ \\
\hline & & $\mathrm{H}$ & btp & $\mathrm{Ch}, \mathrm{O}$ \\
\hline & & $\mathrm{Ar}$ & bmm & $\mathrm{Tr}$ \\
\hline A & NT & $\mathrm{Ab}$ & bc, bmm & $\mathrm{Ne}$ \\
\hline \multirow[t]{7}{*}{ PR } & NT & $\mathrm{Ab}$ & $\mathrm{bc}, \mathrm{bq}, \mathrm{bmm}$ & $\mathrm{Ne}$ \\
\hline & & $\mathrm{Ab}$ & bmm & Ch, B \\
\hline & EN & $\mathrm{Ab}$ & bmm, btp & $\mathrm{Ch}, \mathrm{G}, \mathrm{H}$ \\
\hline & VU & $\mathrm{Ab}$ & bmm & $\mathrm{Ne}$ \\
\hline & EN & $\mathrm{Ab}$ & $\mathrm{bmm}$ & $\mathrm{Ch}, \mathrm{G}$ \\
\hline & & $\mathrm{H}$ & bmm & $\mathrm{Ch}, \mathrm{G}, \mathrm{V}$ \\
\hline & & $\mathrm{Ab}$ & bts & $\mathrm{Ch}, \mathrm{G}, \mathrm{V}$ \\
\hline \multirow[t]{9}{*}{ PR } & & $\mathrm{He}$ & bmm, btp & $\mathrm{Ch}$ \\
\hline & & $\mathrm{He}$ & bmm, btp & $\mathrm{Ch}$ \\
\hline & & $\mathrm{He}$ & btp & $\mathrm{Ch}$ \\
\hline & NT & $\mathrm{Ab}$ & bmm & $\mathrm{Ne}$ \\
\hline & & $\mathrm{Ab}$ & bmm & $\mathrm{Ch}, \mathrm{G}$ \\
\hline & & $\mathrm{Ab}$ & bts & Ch, G \\
\hline & EN & $\mathrm{Ab}$ & bc, bmm & $\mathrm{Ch}, \mathrm{O}$ \\
\hline & VU & $\mathrm{Ab}$ & bc, bmm, btp & $\mathrm{Ch}, \mathrm{G}, \mathrm{O}, \mathrm{S}$ \\
\hline & EN & $\mathrm{Ar}$ & bts & $\mathrm{Ne}$ \\
\hline
\end{tabular}

$\begin{array}{llll} & \text { Ab } & \text { bmm } & \text { Ch, B } \\ & \text { Ab } & \text { bmm } & \text { Ch } \\ & \text { Ab } & \text { bc, bmm, btp } & \text { Ch, S } \\ \text { VU } & \text { Ab } & \text { btp } & \text { Ch } \\ \text { VU } & \text { Ab } & \text { btp } & \text { Ch, V } \\ & \text { Ab } & \text { btp } & \text { Ch, G, O } \\ & \text { Ab } & \text { btp } & \text { Ch, G } \\ \text { CR } & \text { Ab } & \text { bmm } & \text { Ch, G } \\ \text { EN } & \text { Ab } & \text { btp } & \text { Ch, G, T } \\ & & & \\ \text { VU } & & & \text { Ne }\end{array}$


Apéndice 1. Continuación

\begin{tabular}{lllllll}
\hline Grupo/Familia/Especie & NOM & IUCN & HA & TV & DI \\
\hline Licania arborea Seem. & A & & Ab & bts, vs & Ne
\end{tabular}

\section{Chloranthaceae}

Hedyosmum mexicanum C.Cordem.

\section{Clethraceae}

Clethra oleoides L.O.Williams

Clethra parvifolia Lundell

\section{Clusiaceae}

Calophyllum brasiliense var. rekoi (Standl.) Standl.

Clusia guatemalensis Hemsl.

Thornea matudae (Lundell) Breedlove \& E.M.McClint.

\section{Convolvulaceae}

Ipomoea siltepecana Matuda

\section{Cornaceae}

Cornus disciflora DC.

\section{Cunnoniaceae}

Weinmannia pinnata L.

\section{Crassulaceae}

Sedum botterii Hemsl.

\section{Cucurbitaceae}

Sicyos chiriquensis Hammel \& D'Arcy

\section{Ebenaceae}

Diospyros morenoi A.Pool

Elaeocarpaceae

Sloanea terniflora (Sessé \& Moc. ex DC.) Standl.

\section{Ericaceae}

Comarostaphylis arbutoides Lindl. subsp. arbutoides

Vaccinium leucanthum Schltdl.

\section{Euphorbiaceae}

Acalypha chiapensis Brandegee

Acalypha macrostachyoides Muell.Arg.

Bernardia oblanceolata Lundell

Croton chiapensis Lundell

Croton guatemalensis Lotsy

Croton siltepecensis Lundell

Phyllanthus purpusii Brandegee

Tetrorchidium rotundatum Standl

VU $\quad \mathrm{Ab} \quad \mathrm{bmm} \quad \mathrm{Ne}$

VU $\quad A b \quad b m m \quad C h, G, S$

$\mathrm{Ab} \quad \mathrm{bmm} \quad \mathrm{Tr}$

A

EN

Ab bc, btc, bts $\quad \mathrm{Ne}$

$\mathrm{Ab} \quad$ bc, bq, bmm, btp $\mathrm{Ne}$

Ar bmm

Ch, N

VU

Ab bc, bmm

$\mathrm{Ne}$

NT

Ab bmm, btp, vs

$\mathrm{Ne}$

$\mathrm{He} \quad \mathrm{bc}$

Ch, V

B $\quad b m m$

Ch, CR, P

Ab btp

Ch, N, S

Ab btc, btp

$\mathrm{Ne}$

VU $\quad A b \quad b m m \quad \mathrm{Ne}$

$\begin{array}{llll}\text { EN } & \mathrm{Ar} & \mathrm{bmm} & \mathrm{Ne}\end{array}$

$\begin{array}{llll} & \text { Ar } & \text { bc, bq, bmm, btp } & \text { Ch } \\ \text { EN } & \text { Ar } & \text { btp } & \text { Ch, V } \\ & \text { Ab } & \text { bmm } & \text { Ch, G, O } \\ & \text { Ar } & \text { btp } & \text { Tr } \\ \text { Ab } & \text { bc, btc, btp } & \mathrm{Ne} \\ \text { Ar } & \text { bmm } & \text { Ch } \\ \text { Ar } & \text { bmm } & \text { Ch, G } \\ \text { Ab } & \text { btp } & \text { Ne }\end{array}$


Apéndice 1. Continuación

\begin{tabular}{|c|c|c|c|c|c|}
\hline Grupo/Familia/Especie & NOM & IUCN & HA & TV & DI \\
\hline \multicolumn{6}{|l|}{ Fabaceae } \\
\hline Acosmium panamense (Benth.) Yakovlev & $A$ & & $\mathrm{Ab}$ & btp & $\mathrm{Ne}$ \\
\hline Ateleia pterocarpa Moc. \& Sessé ex D.Dietr. & & NT & $\mathrm{Ab}$ & bc & $\mathrm{Ne}$ \\
\hline Calopogonium lanceolatum Brandegee & & & B & bmm & Ch, G \\
\hline Canavalia dura J.D.Sauer & & & B & bc, bmm & $\mathrm{Ch}, \mathrm{Gu}$ \\
\hline Canavalia matudae J.D.Sauer & & & $\mathrm{B}$ & $\mathrm{bc}, \mathrm{bmm}$ & $\mathrm{Ch}$ \\
\hline Cojoba arborea (L.) Britton \& Rose & & NT & $\mathrm{Ab}$ & bmm & $\mathrm{Ne}$ \\
\hline Cojoba escuintlensis (Lundell) L.Rico & & EN & $\mathrm{Ab}$ & btp & $\mathrm{Ch}, \mathrm{O}, \mathrm{T}$ \\
\hline Cojoba matudae (Lundell) L.Rico & & VU & $\mathrm{Ab}$ & bmm & $\mathrm{Ch}, \mathrm{Gu}, \mathrm{O}$ \\
\hline Cojoba siltepecensis (Lundell) L.Rico & & & $\mathrm{Ab}$ & bmm & $\mathrm{Ch}$ \\
\hline Desmodium chiapense Brandegee & & & B & btp & $\mathrm{Ch}$ \\
\hline Desmodium helleri Peyr. & & & B & bc, bmm, btp, vs & $\mathrm{Ch}, \mathrm{O}, \mathrm{V}$ \\
\hline Erythrina goldmanii Standl. & & & $\mathrm{Ab}$ & btc, btp & $\mathrm{Ch}, \mathrm{O}$ \\
\hline Eysenhardtia adenostylis Baill. & & & $\mathrm{Ab}$ & bc, btc & $\mathrm{Ch}, \mathrm{G}, \mathrm{H}, \mathrm{S}$, \\
\hline Indigofera matudae Lundell & & & $\mathrm{H}$ & bmm & $\mathrm{Ch}$ \\
\hline Inga calderonii Standl. & & CR & $\mathrm{Ab}$ & bc, bts & $\mathrm{Ch}, \mathrm{G}, \mathrm{S}$ \\
\hline Inga flexuosa Schltdl. & & CR & $\mathrm{Ab}$ & bmm, btp & $\mathrm{Ne}$ \\
\hline Inga stenophylla Standl. & & EN & $\mathrm{Ab}$ & btp & $\mathrm{Ne}$ \\
\hline Leucaena leucocephala subsp. ixtahuacana C.E. Hughes & & & $\mathrm{Ab}$ & btp & Ch, G \\
\hline Lonchocarpus minimiflorus Donn.Sm. & & EN & $\mathrm{Ab}$ & btp & $\mathrm{Ne}$ \\
\hline Mimosa velloziana var. maxonii (Standl.) R.Grether & & & $\mathrm{H}$ & btp & Ch, G \\
\hline Vatairea lundellii (Standl.) Killip ex Record & $\mathrm{P}$ & & $\mathrm{Ab}$ & bts & $\mathrm{Ne}$ \\
\hline \multicolumn{6}{|l|}{ Fagaceae } \\
\hline Quercus benthamii A.DC. & & EN & $\mathrm{Ab}$ & bmm & $\mathrm{Ne}$ \\
\hline Quercus candicans Née & & VU & $\mathrm{Ab}$ & bc, bq, bmm, btp & $\mathrm{Ne}$ \\
\hline Quercus corrugata Hook. & & EN & $\mathrm{Ab}$ & bmm, btp & $\mathrm{Ne}$ \\
\hline Quercus cortesii Liebm. & & NT & $\mathrm{Ab}$ & bc & $\mathrm{Ne}$ \\
\hline Quercus elliptica Née & & VU & $\mathrm{Ab}$ & bc, bq, bmm, bts & $\mathrm{Ne}$ \\
\hline Quercus insignis M.Martens \& Galeotti & & CR & $\mathrm{Ab}$ & bc & $\mathrm{Ne}$ \\
\hline Quercus lancifolia Schltdl. \& Cham. & & NT & $\mathrm{Ab}$ & bmm & $\mathrm{Ne}$ \\
\hline Quercus paxtalensis C.H.Muller & & CR & $\mathrm{Ab}$ & bmm & $\mathrm{Ne}$ \\
\hline Quercus sapotifolia Liebm. & & VU & $\mathrm{Ab}$ & bc, bmm & $\mathrm{Ne}$ \\
\hline Quercus sartorii Liebm. & & EN & $\mathrm{Ab}$ & bmm & $\mathrm{Ne}$ \\
\hline Quercus segoviensis Liebm. & & VU & $\mathrm{Ab}$ & bmm & $\mathrm{Ne}$ \\
\hline Quercus skinneri Benth. & & CR & $\mathrm{Ab}$ & bc, bq, bmm, btp & $\mathrm{Ne}$ \\
\hline Quercus vicentensis Trel. & & VU & $\mathrm{Ab}$ & btp & $\mathrm{Ne}$ \\
\hline \multicolumn{6}{|l|}{ Flacourtiaceae } \\
\hline Casearia arguta Kunth & & NT & $\operatorname{Ar}$ & bmm, btp & $\mathrm{Ne}$ \\
\hline Casearia tacanensis Lundell & & NT & $\operatorname{Ar}$ & bmm, btp & $\mathrm{Ne}$ \\
\hline Hasseltia guatemalensis Warb. & & EN & $\operatorname{Ar}$ & btp & $\mathrm{Ne}$ \\
\hline Lunania mexicana Brandegee & & VU & $\mathrm{Ab}$ & bmm & $\mathrm{Ne}$ \\
\hline Olmediella betschleriana (Göpp.) Loes. & & EN & $\mathrm{Ab}$ & bc, bmm, btp & $\mathrm{Ne}$ \\
\hline \multicolumn{6}{|l|}{ Gentianaceae } \\
\hline Lisianthius brevidentatus (Hemsl.) Kuntze & & & $\mathrm{H}$ & bq, va & $\mathrm{Ch}, \mathrm{B}, \mathrm{G}$ \\
\hline Lisianthius orepolus B.L.Rob. & & & $\mathrm{H}$ & bc, vs & $\mathrm{Ch}$ \\
\hline
\end{tabular}


Apéndice 1. Continuación

\begin{tabular}{|c|c|c|c|c|c|}
\hline Grupo/Familia/Especie & NOM & IUCN & HA & TV & DI \\
\hline \multicolumn{6}{|l|}{ Gesneriaceae } \\
\hline Achimenes candida Lindl. & & & $\mathrm{H}$ & bq, bmm, btp & $\mathrm{Ch}, \mathrm{CR}, \mathrm{G}, \mathrm{P}$ \\
\hline Achimenes cettoana H.E.Moore & & & $\mathrm{H}$ & bmm & $\mathrm{Ch}$ \\
\hline Achimenes misera Lindl. & & & $\mathrm{H}$ & bc, bq, bmm, btp & $\mathrm{Ch}, \mathrm{G}, \mathrm{H}, \mathrm{S}$ \\
\hline Kohleria rugata (Scheidw.) L.P.Kvist \& L.E.Skog & & & $\mathrm{H}$ & bc, bts & $\mathrm{Ch}, \mathrm{Gu}$ \\
\hline Neomortonia nummularia (Hanst.) Wiehler & & & $\mathrm{H}$ & bmm, btp & $\mathrm{Ch}, \mathrm{G}$ \\
\hline Niphaea saxifola (Brandegee) D.N.Gibson & & & $\mathrm{H}$ & bc, bmm & $\mathrm{Ch}$ \\
\hline Solenophora glomerata Weigend \& Forther & & & $\mathrm{H}$ & bmm & $\operatorname{Tr}$ \\
\hline Solenophora purpusii Brandegee & & & $\operatorname{Ar}$ & bmm, btp, bts & Ch, G \\
\hline \multicolumn{6}{|l|}{ Hammamelidaceae } \\
\hline Matudaea trinervia Lundell & A & VU & $\mathrm{Ab}$ & bmm & $\mathrm{Ne}$ \\
\hline \multicolumn{6}{|l|}{ Lamiaceae } \\
\hline Catopheria chiapensis A.Gray ex Benth. & & & $\mathrm{Ar}$ & bc & $\mathrm{Ch}, \mathrm{G}, \mathrm{H}, \mathrm{S}$ \\
\hline Chaunostoma mecistandrum Donn.Sm. & & & $\mathrm{Ar}$ & bmm & $\mathrm{Ch}, \mathrm{G}$ \\
\hline Salvia curtiflora Epling & & & $\mathrm{H}$ & bmm & $\mathrm{Ch}, \mathrm{G}$ \\
\hline Salvia eizi-matudae Ramamoorthy & & & $\mathrm{H}$ & bmm & Ch \\
\hline Salvia miniata Fernald & & & $\mathrm{H}$ & btp & $\mathrm{Ch}, \mathrm{B}$ \\
\hline Salvia rubiginosa Benth. & & & $\mathrm{H}$ & bc, bmm, btp, bts & $\mathrm{Ch}, \mathrm{O}, \mathrm{V}$ \\
\hline Salvia tonalensis Brandegee & & & $\operatorname{Ar}$ & bc & $\mathrm{Ch}$ \\
\hline Scutellaria guatemalensis Leonard & & & $\mathrm{H}$ & bc & $\mathrm{Ch}, \mathrm{G}$ \\
\hline Scutellaria lundellii Epling & & & $\mathrm{H}$ & btp & $\mathrm{Ch}, \mathrm{B}$ \\
\hline Scutellaria orizabensis Epling & & & $\mathrm{H}$ & bmm & Ch, V \\
\hline Scutellaria vitifolia Brandegee & & & $\mathrm{H}$ & bmm & Ch, G \\
\hline \multicolumn{6}{|l|}{ Lauraceae } \\
\hline Cinnamomum areolatum (Lundell) Kosterm. & & EN & $\mathrm{Ab}$ & bc, bmm, btp & $\mathrm{Ch}, \mathrm{O}, \mathrm{S}$ \\
\hline Cinnamomum chiapense (Lundell) Kosterm. & & & $\mathrm{Ab}$ & bmm & $\operatorname{Tr}$ \\
\hline Licaria campechiana (Standl.) Kosterm. & & $\mathrm{VU}$ & $\mathrm{Ab}$ & bmm, btp & $\mathrm{Ne}$ \\
\hline Licaria excelsa Kosterm. & & VU & $\mathrm{Ab}$ & $\mathrm{bmm}$ & $\mathrm{Ne}$ \\
\hline Licaria glaberrima (Lundell) C.K.Allen & & EN & $\mathrm{Ab}$ & bmm & $\mathrm{Ch}$ \\
\hline Litsea glaucescens Kunth & $\mathrm{P}$ & VU & $\mathrm{Ab}$ & bc, bmm & $\mathrm{Ne}$ \\
\hline Nectandra cuspidata Nees & & NT & $\mathrm{Ab}$ & bmm & $\mathrm{Ne}$ \\
\hline Nectandra hypoleuca Hammel & & $\mathrm{VU}$ & $\mathrm{Ab}$ & btp & $\mathrm{Ne}$ \\
\hline Nectandra matudae Lundell & & VU & $\mathrm{Ab}$ & bmm & $\mathrm{Ch}$ \\
\hline Nectandra reticulata (Ruiz \& Pav.) Mez & & NT & $\mathrm{Ab}$ & btp, vs & $\mathrm{Ne}$ \\
\hline Nectandra rudis C.K.Allen & & EN & $\mathrm{Ab}$ & bmm & $\mathrm{Ch}, \mathrm{G}, \mathrm{S}$ \\
\hline Nectandra salicifolia (Kunth) Nees & & NT & $\mathrm{Ab}$ & bmm & $\mathrm{Ne}$ \\
\hline Nectandra salicina C.K.Allen & & EN & $\mathrm{Ab}$ & btp & $\mathrm{Ch}, \mathrm{CR}, \mathrm{P}$ \\
\hline Ocotea acuminatissima (Lundell) Rohwer & & VU & $\mathrm{Ab}$ & bc, bmm, btp & $\mathrm{Ch}, \mathrm{G}, \mathrm{Gu}, \mathrm{O}$ \\
\hline Ocotea botrantha Rohwer & & EN & $\mathrm{Ab}$ & bq, bmm, btp & $\mathrm{Ne}$ \\
\hline Ocotea chiapensis (Lundell) Standl. \& Steyerm. & & EN & $\mathrm{Ab}$ & bmm & $\mathrm{Ch}, \mathrm{G}, \mathrm{Gu}, \mathrm{O}$ \\
\hline Ocotea helicterifolia (Meisn.) Hemsl. & & VU & $\mathrm{Ab}$ & bmm & $\mathrm{Ne}$ \\
\hline Ocotea leucoxylon (Sw.) Laness. & & NT & $\mathrm{Ab}$ & bmm & $\mathrm{Ne}$ \\
\hline Ocotea matudae Lundell & & EN & $\mathrm{Ab}$ & bmm & $\operatorname{Tr}$ \\
\hline Ocotea platyphylla (Lundell) Rohwer & & EN & $\mathrm{Ab}$ & bmm & $\mathrm{Ch}, \mathrm{G}, \mathrm{S}$ \\
\hline Ocotea sinuata (Mez) Rohwer & & VU & $\mathrm{Ab}$ & btc, bts & $\mathrm{Ne}$ \\
\hline Persea americana Mill. & & EN & $\mathrm{Ab}$ & bmm, btp & $\mathrm{Ne}$ \\
\hline Persea donnell-smithii Mez & & VU & $\mathrm{Ab}$ & btp & $\mathrm{Ne}$ \\
\hline
\end{tabular}


Apéndice 1. Continuación

\begin{tabular}{l} 
Grupo/Familia/Especie \\
\hline Persea liebmannii Mez \\
Persea schiedeana Nees \\
Loasaceae \\
Schismocarpus matudae Steyerm. \\
Loganiaceae \\
Buddleja cordata subsp. ovandensis (Lundell \\
ex E.M.Norman) E.M.Norman \\
Buddleja skutchii C.V.Morton
\end{tabular}

\section{Loranthaceae}

Struthanthus capitatus Lundell

Struthanthus matudae Lundell

\section{Lythraceae}

Cuphea subuligera Koehne

\section{Malpighiaceae}

Bunchosia matudae Lundell

Mascagnia dipholiphylla (Small) Bullock

\section{Malvaceae}

Bakeridesia nelsonii (Rose) D.M.Bates

Pavonia monticola Fryxell

Robinsonella samaricarpa Fryxell

\section{Melastomataceae}

Conostegia volcanalis Standl. \& Steyerm.

Heterocentron muricatum Gleason

Miconia alpestris Cogn

Miconia chinantlana (Naudin) Almeda

Miconia mexicana (Bonpl.) Naudin

Tibouchina sericea R.deSantiago

\section{Meliaceae}

Guarea glabra Vahl

Cedrela odorata L.

\section{Menispermaceae}

Abuta chiapasensis Krukoff \& Barneby

\section{Mitrastemonaceae}

Mitrastemon matudae Yamam.

\section{Monimiaceae}

Mollinedia pallida Lundell

\begin{tabular}{|c|c|c|c|c|}
\hline NOM & IUCN & HA & TV & DI \\
\hline & NT & $\mathrm{Ab}$ & bmm, btp & $\mathrm{Ch}, \mathrm{G}$ \\
\hline & \multirow[t]{2}{*}{ VU } & $\mathrm{Ab}$ & btp & $\mathrm{Ne}$ \\
\hline & & $\mathrm{H}$ & btp, bts & $\mathrm{Ch}$ \\
\hline & EN & $\mathrm{Ab}$ & bmm & $\mathrm{Tr}$ \\
\hline & VU & $\mathrm{Ab}$ & bmm & $\mathrm{Ch}, \mathrm{Cr}, \mathrm{G}, \mathrm{P}$ \\
\hline & & B & bmm & $\mathrm{Ch}$ \\
\hline & & B & btp & $\mathrm{Ch}, \mathrm{G}$ \\
\hline & & $\mathrm{H}$ & bc, bmm, btp, vs & $\mathrm{Ch}$ \\
\hline & & $\mathrm{Ab}$ & bmm & $\mathrm{Ch}, \mathrm{G}$ \\
\hline & & B & btp & $\mathrm{Ch}, \mathrm{O}, \mathrm{V}$ \\
\hline & & $\mathrm{Ar}$ & bts & $\mathrm{Ch}, \mathrm{G}$ \\
\hline & & $\mathrm{Ar}$ & bts & $\mathrm{Ch}, \mathrm{O}$ \\
\hline & VU & $\mathrm{Ar}$ & bts & $\mathrm{Ne}$ \\
\hline & VU & $\mathrm{Ar}$ & bmm & $\mathrm{Ne}$ \\
\hline & & $\mathrm{H}$ & bts & $\mathrm{Ch}$ \\
\hline & & $\mathrm{Ar}$ & bmm & $\mathrm{Ch}, \mathrm{G}$ \\
\hline & CR & $\mathrm{Ar}$ & btp & $\mathrm{Ch}, \mathrm{G}, \mathrm{O}$ \\
\hline & VU & $\mathrm{Ar}$ & bc, bmm, btp & $\mathrm{Ne}$ \\
\hline & & $\mathrm{Ar}$ & bmm & $\mathrm{Ch}, \mathrm{Gu}, \mathrm{O}$ \\
\hline & NT & $\mathrm{Ab}$ & bmm, btp & $\mathrm{Ne}$ \\
\hline \multirow[t]{4}{*}{ PR } & \multirow[t]{3}{*}{ VU } & $\mathrm{Ab}$ & btp, vs & $\mathrm{Ne}$ \\
\hline & & B & bts & Ch, G \\
\hline & & $\mathrm{He}$ & bmm, vs & Ch, G \\
\hline & EN & $\mathrm{Ab}$ & $\begin{array}{l}\text { bc, bmm, btp, } \\
\text { bts, vs }\end{array}$ & $\mathrm{Ch}, \mathrm{G}$ \\
\hline
\end{tabular}


Apéndice 1. Continuación

\begin{tabular}{|c|c|c|c|c|c|}
\hline Grupo/Familia/Especie & NOM & IUCN & HA & TV & DI \\
\hline \multicolumn{6}{|l|}{ Moraceae } \\
\hline Ficus cookii Standl. & & & $\mathrm{Ab}$ & bmm, btc, btp & $\mathrm{Ch}, \mathrm{G}, \mathrm{V}$ \\
\hline Trophis cuspidata Lundell & & $\mathrm{VU}$ & $\mathrm{Ab}$ & bmm & $\mathrm{Ch}, \mathrm{G}, \mathrm{O}$ \\
\hline \multicolumn{6}{|l|}{ Myrsinaceae } \\
\hline Ardisia breedlovei Lundell & & EN & $\mathrm{Ab}$ & bmm & $\mathrm{Ch}$ \\
\hline Ardisia liebmannii Oerst. & & $\mathrm{VU}$ & $\mathrm{Ab}$ & $\mathrm{bmm}$ & $\mathrm{Ne}$ \\
\hline $\begin{array}{l}\text { Ardisia mexicana subsp. siltepecana (Lundell) } \\
\text { Pipoly \& Ricketson }\end{array}$ & & EN & $\mathrm{Ab}$ & bmm & $\mathrm{Ch}$ \\
\hline Ardisia revoluta Kunth & & NT & $A b$ & bc, btp & $\mathrm{Ne}$ \\
\hline $\begin{array}{l}\text { Ardisia verapazensis subsp. cucullata (Lundell) } \\
\text { Pipoly \& Ricketson }\end{array}$ & & & $\mathrm{Ab}$ & bmm, btp & $\mathrm{Ch}$ \\
\hline Ctenardisia purpusii (Brandegee) Lundell & & EN & $A b$ & bmm & $\mathrm{Ch}$ \\
\hline Gentlea tacanensis (Lundell) Lundell & & EN & $\mathrm{Ab}$ & bmm, btp & $\mathrm{Ch}, \mathrm{G}$ \\
\hline Myrsine juergensenii (Mez) Ricketson \& Pipoly & & NT & $\mathrm{Ab}$ & bc, bmm & $\mathrm{Ne}$ \\
\hline Parathesis chiapensis Fernald & & $\mathrm{VU}$ & $\mathrm{Ab}$ & bc, bq, bmm, btp & $\mathrm{Ch}$ \\
\hline Parathesis columnaris Lundell & & EN & $\mathrm{Ab}$ & bmm & $\mathrm{Ch}, \mathrm{G}$ \\
\hline Parathesis donnell-smithii Mez & & NT & $\mathrm{Ab}$ & bmm & $\mathrm{Ne}$ \\
\hline Parathesis lanceolata Brandegee & & EN & $\mathrm{Ab}$ & btp & $\mathrm{Ch}, \mathrm{G}$ \\
\hline Parathesis leptopa Lundell & & EN & $\mathrm{Ab}$ & bmm & $\mathrm{Ne}$ \\
\hline Parathesis multiflora Lundell & & & $\mathrm{Ab}$ & btp & $\mathrm{Ch}$ \\
\hline Parathesis nigropunctata Lundell & & & $A b$ & bmm, btp & $\mathrm{Ch}$ \\
\hline Parathesis subcoriacea Lundell & & EN & $\mathrm{Ab}$ & bmm, btp, bts & $\mathrm{Ch}, \mathrm{G}$ \\
\hline Parathesis subulata Lundell & & EN & $\mathrm{Ab}$ & bmm & $\mathrm{Ch}, \mathrm{G}$ \\
\hline Parathesis vulgata Lundell & & EN & $\mathrm{Ab}$ & bmm & $\mathrm{Ch}, \mathrm{G}, \mathrm{H}, \mathrm{S}$ \\
\hline Synardisia venosa (Mast.) Lundell & & NT & $\mathrm{Ab}$ & bmm & $\mathrm{Ne}$ \\
\hline Yunckeria ovandensis (Lundell) Lundell & & & $\mathrm{Ab}$ & bc, bmm, btp & $\mathrm{Ch}, \mathrm{G}$ \\
\hline \multicolumn{6}{|l|}{ Myrtaceae } \\
\hline Calyptranthes chiapensis Lundell & & & $\operatorname{Ar}$ & bts & $\mathrm{Ch}, \mathrm{V}$ \\
\hline Calyptranthes perlaevigata Lundell & & & $\mathrm{Ab}$ & bmm & $\mathrm{Ch}$ \\
\hline Eugenia amatenangensis Lundell & & & $\mathrm{Ab}$ & btp, bts & $\mathrm{Ch}$ \\
\hline Eugenia breedlovei Barrie & & & $\mathrm{Ab}$ & bmm & $\mathrm{Ch}$ \\
\hline Eugenia capulioides Lundell & & & $\mathrm{Ab}$ & vs & $\mathrm{Ch}, \mathrm{G}$ \\
\hline Eugenia chiapensis Lundell & & & $\mathrm{Ab}$ & bmm & $\mathrm{Ch}$ \\
\hline Eugenia citroides Lundell & & EN & $\mathrm{Ab}$ & bmm & $\mathrm{Ch}, \mathrm{G}$ \\
\hline Eugenia letreroana Lundell & & EN & $\mathrm{Ab}$ & bmm & Ch, G \\
\hline Eugenia matudae Lundell & & & $\mathrm{Ab}$ & bmm & $\mathrm{Ch}$ \\
\hline Eugenia mexicana Steud. & & $\mathrm{VU}$ & $\mathrm{Ab}$ & bmm & $\mathrm{Ne}$ \\
\hline Eugenia nigrita Lundell & & & $\mathrm{Ab}$ & bmm & $\mathrm{Ch}$ \\
\hline Eugenia ovandensis Lundell & & CR & $\mathrm{Ab}$ & bmm & $\operatorname{Tr}$ \\
\hline Eugenia siltepecana Lundell & & & $\mathrm{Ab}$ & bc, btp & $\mathrm{Ch}$ \\
\hline Eugenia uliginosa Lundell & & & $\mathrm{Ab}$ & bmm, btp & $\mathrm{Ch}$ \\
\hline \multicolumn{6}{|l|}{ Nyctaginaceae } \\
\hline Neea turbinata Lundell & & & $\mathrm{Ab}$ & bmm & $\mathrm{Ch}$ \\
\hline \multicolumn{6}{|l|}{ Ochnaceae } \\
\hline Ouratea tuerckheimii Donn.Sm. & & & $A b$ & bc, btp & $\mathrm{Ch}, \mathrm{G}, \mathrm{V}$ \\
\hline
\end{tabular}


Apéndice 1. Continuación

\section{Grupo/Familia/Especie}

\section{Oleaceae}

Osmanthus americanus (L.) Benth. \& Hook.f. ex A.Gray

Osmanthus mexicanus Lundell

\section{Onagraceae}

Fuchsia microphylla subsp. aprica (Lundell) Breedlove

\section{Oxalidaceae}

Oxalis dimidiata Donn.Sm.

\section{Passifloraceae}

Passiflora apetala Killip

Passiflora dolichocarpa Killip

Passiflora ornithoura var. chiapasensis J.M.MacDougal

Passiflora prolata Mast.

Passiflora sanctae-mariae J.M.MacDougal

Phyllonomaceae

Phyllonoma laticuspis (Turcz.) Engl.

\section{Piperaceae}

Peperomia guatemalensis C.DC.

Piper vergelense Trel. \& Standl.

\section{Platanaceae}

Platanus mexicana Moric.

\section{Polemoniaceae}

Cobaea biaurita Standl.

Cobaea pachysepala Standl.

\section{Polygalaceae}

Monnina guatemalensis Chodat

\section{Poligonaceae}

Coccoloba matudae Lundell

\section{Proteaceae}

Roupala montana Aubl.

\section{Rhamnaceae}

Rhamnus sphaerosperma Sw. var. mesoamericana

M.C.Johnst. \& L.A.Johnst.

\section{Rosaceae}

Photinia matudae Lundell

Prunus barbata Koehne

Prunus brachybotrya Zucc.

Prunus lundelliana Standl.
VU

NT

$\begin{array}{lll}\mathrm{Ab} & \text { bmm, btp } & \mathrm{Ne} \\ \mathrm{Ar} & \text { btp } & \mathrm{Ch}, \mathrm{O}\end{array}$

Ar bmm

Ch, G, H, S

H btp

Ch, G

VU

Ar bc, bmm

$\mathrm{Ne}$

He bmm

Ch, B

Ar bmm

Ch, G

Ab bmm, btp, va $\mathrm{Ne}$

$\begin{array}{lll}\mathrm{H} & \text { bmm } & \mathrm{Ch}, \mathrm{O}, \mathrm{V} \\ \mathrm{H} & \text { bmm } & \text { Ch, G }\end{array}$

$\mathrm{Ar} \quad \mathrm{bmm}$

Ch, B

$\mathrm{Ab} \quad$ btp

Ch, O

$\mathrm{Ab}$

btp, bts, vs

$\mathrm{Ne}$

VU

$\mathrm{Ab}$

bmm

$\mathrm{Ne}$

CR

EN

VU

EN

$\begin{array}{lll}\mathrm{Ab} & \text { bmm } & \mathrm{Ch}, \mathrm{G} \\ \mathrm{Ab} & \text { bmm } & \mathrm{Ch}, \mathrm{G} \\ \mathrm{Ab} & \text { bmm, btp, bts } & \mathrm{Ne} \\ \mathrm{Ab} & \text { bmm } & \mathrm{Ne}\end{array}$


Apéndice 1. Continuación

\begin{tabular}{|c|c|c|c|c|c|}
\hline Grupo/Familia/Especie & NOM & IUCN & HA & TV & DI \\
\hline \multicolumn{6}{|l|}{ Rubiaceae } \\
\hline Arachnothryx armentalis (L.O.Williams) Lorence & & & $\mathrm{Ab}$ & btp & $\mathrm{Ch}, \mathrm{G}, \mathrm{O}$ \\
\hline Arachnothryx buddleioides (Benth.) Planch. & & $\mathrm{VU}$ & $\mathrm{Ab}$ & bmm, btp & $\mathrm{Ne}$ \\
\hline Arachnothryx flocculosa Borhidi & & & $\mathrm{Ab}$ & bmm & $\mathrm{Ch}$ \\
\hline Arachnothryx laniflora (Benth.) Planch. & & $\mathrm{VU}$ & $\mathrm{Ab}$ & bc, bmm & $\mathrm{Ne}$ \\
\hline Arachnothryx pauciflora Borhidi & & $\mathrm{CR}$ & $\mathrm{Ab}$ & bmm & $\operatorname{Tr}$ \\
\hline Arachnothryx pyramidalis (Lundell) Borhidi & & $\mathrm{VU}$ & $\mathrm{Ab}$ & bmm & $\mathrm{Ch}, \mathrm{Gu}, \mathrm{O}$ \\
\hline Balmea stormae Martínez & $\mathrm{PR}$ & EN & $\mathrm{Ab}$ & bmm & $\mathrm{Ne}$ \\
\hline Bouvardia dictyoneura Standl. & PR & & $\mathrm{H}$ & bmm & $\mathrm{Ch}, \mathrm{G}$ \\
\hline Bouvardia quinquenervata Standl. & & & $\operatorname{Ar}$ & btc, btp & $\mathrm{Ch}$ \\
\hline Chomelia brachypoda Donn.Sm. & & EN & $\mathrm{Ab}$ & bmm & $\mathrm{Ch}, \mathrm{G}, \mathrm{O}$ \\
\hline Chomelia protracta (Bart. ex DC.) Standl. & & VU & $\mathrm{Ab}$ & bmm & $\mathrm{Ne}$ \\
\hline Deppea erythrorhiza Cham. \& Schltdl. & & & $\mathrm{Ar}$ & btp & $\mathrm{Ch}, \mathrm{O}$ \\
\hline Deppea grandiflora Schltdl. & & $\mathrm{VU}$ & $\mathrm{Ab}$ & btp & $\mathrm{Ne}$ \\
\hline Deppea inaequalis Standl. \& Steyerm. & & & $\mathrm{Ar}$ & btp & $\mathrm{Ch}, \mathrm{CR}, \mathrm{G}, \mathrm{H}$ \\
\hline Glossostipula concinna (Standl.) Lorence & & EN & $\mathrm{Ab}$ & bc, bq, bmm, btp & $\mathrm{Ne}$ \\
\hline Gonzalagunia chiapensis (Standl.) Standl. \& Steyerm. & & EN & $\mathrm{Ab}$ & btp & $\mathrm{Ne}$ \\
\hline Hamelia barbata Standl. & & CR & $\mathrm{Ar}$ & bts & $\mathrm{Ch}, \mathrm{G}$ \\
\hline Hillia macrocarpa Standl. \& Steyerm. & & & $\mathrm{H}$ & bc & $\mathrm{Ch}, \mathrm{G}$ \\
\hline Hintonia lumaeana (Baill.) Bullock & & & $\mathrm{Ar}$ & bmm & Ch, CR, G, S \\
\hline Hoffmannia macrosiphon Standl. & & & $\operatorname{Ar}$ & bmm & Ch, G \\
\hline Hoffmannia quadrifolia Standl. \& Steyerm. & & & $\operatorname{Ar}$ & bmm & $\mathrm{Ch}, \mathrm{G}$ \\
\hline Hoffmannia steyermarkii Standl. & & & $\mathrm{Ar}$ & bmm & $\mathrm{Ch}, \mathrm{G}$ \\
\hline Pseudomiltemia filisepala (Standl.) Borhidi & PR & & $\mathrm{Ar}$ & bc, bq, bmm & $\mathrm{Ch}, \mathrm{Gu}$ \\
\hline Pseudomiltemia davidsonii Martínez-Camilo \& Lorence & & & $\operatorname{Ar}$ & bmm & $\operatorname{Tr}$ \\
\hline Pinarophyllon flavum Brandegee & PR & & $\mathrm{H}$ & btc, btp, bts, vs & $\mathrm{Ch}, \mathrm{G}$ \\
\hline Plocaniophyllon flavum Brandegee & & & $\mathrm{H}$ & btp, bts & $\mathrm{Ch}, \mathrm{G}$ \\
\hline Psychotria chiapensis Standl. & & VU & $\mathrm{Ab}$ & bmm & $\mathrm{Ne}$ \\
\hline Psychotria galeottiana (M.Martens) C.M.Taylor \& Lorence & & $\mathrm{VU}$ & $\mathrm{Ab}$ & bc, bmm & $\mathrm{Ne}$ \\
\hline Psychotria panamensis Standl. var. panamensis & & $\mathrm{VU}$ & $\mathrm{Ab}$ & bmm & $\mathrm{Ne}$ \\
\hline Psychotria purpusii Standl. & & & $\mathrm{Ar}$ & bmm & $\mathrm{Ch}, \mathrm{G}$ \\
\hline Rogiera amoena Planch. & & $\mathrm{VU}$ & $\mathrm{Ab}$ & bc, bmm, btp, vs & $\mathrm{Ne}$ \\
\hline Rogiera cordata (Benth.) Planch. & & EN & $\mathrm{Ab}$ & bc, bmm, btp & $\mathrm{Ch}, \mathrm{G}, \mathrm{O}$ \\
\hline Rogiera suffrutescens (Brandegee) Borhidi & & & $\operatorname{Ar}$ & bmm & $\mathrm{Ch}, \mathrm{G}$ \\
\hline Sommera arborescens Schltdl. & & EN & $\operatorname{Ar}$ & bmm & $\mathrm{Ch}, \mathrm{G}, \mathrm{O}, \mathrm{V}$ \\
\hline \multicolumn{6}{|l|}{ Rutaceae } \\
\hline Zanthoxylum harmsianum (Loes.) P.Wilson & & & $\mathrm{Ab}$ & bc & $\mathrm{Ch}, \mathrm{B}$ \\
\hline Zanthoxylum procerum Donn.Sm. & & EN & $\mathrm{Ab}$ & btc & $\mathrm{Ne}$ \\
\hline \multicolumn{6}{|l|}{ Sabiaceae } \\
\hline Meliosma matudae Lundell & & & $\mathrm{Ab}$ & bmm & $\operatorname{Tr}$ \\
\hline \multicolumn{6}{|l|}{ Sapindaceae } \\
\hline Cupania mollis Standl. & & & $\mathrm{Ab}$ & bc & $\mathrm{Ch}, \mathrm{B}$ \\
\hline Cupania prisca Standl. & & & $\mathrm{Ab}$ & btp & $\mathrm{Ch}, \mathrm{G}$ \\
\hline \multicolumn{6}{|l|}{ Sapotaceae } \\
\hline Sideroxylon capiri (A.DC.) Pittier & A & & $\mathrm{Ab}$ & btp & $\mathrm{Ne}$ \\
\hline
\end{tabular}


Apéndice 1. Continuación

\begin{tabular}{lllllll}
\hline Grupo/Familia/Especie & NOM & IUCN & HA & TV & DI \\
\hline Sideroxylon portoricense Urb. subsp. minutiflorum & & VU & Ab & btp & Ne
\end{tabular}

(Pittier) T.D.Penn.

\section{Scrophulariaceae}

Castilleja chiapensis Brandegee

\section{Simaroubaceae}

Picramnia matudae Lundell

\section{Solanaceae}

Cestrum elegantissimum C.V.Morton

Cestrum guatemalense Francey

Cestrum regelii Planch.

Lycianthes connata J.L.Gentry

Solanum oaxacanum Dunal

Tzeltalia amphitricha (Bitter) E.Estrada \& M.Martínez

\section{Styracaceae}

Styrax glabrescens Benth.

\section{Symplocaceae}

$\mathrm{H} \quad$ bc

$\mathrm{Ch}, \mathrm{Gu}$

Ar bc, bmm

Ch

$\begin{array}{lll}\text { Ar } & \text { bc, bq, bmm, } & \text { Ch, G } \\ & \text { bts, vs } & \\ \mathrm{Ar} & \text { bmm } & \text { Ch, G, S } \\ \mathrm{Ar} & \text { bmm } & \text { Ch, G } \\ \mathrm{Ar} & \text { bmm } & \text { Ch, B } \\ \mathrm{Ar} & \text { btp } & \text { Ch, O } \\ \mathrm{Ar} & \text { bmm } & \text { Ch, G }\end{array}$

$\mathrm{Ab} \quad$ bc, bmm, btc, vs $\mathrm{Ne}$

Symplocos breedlovei Lundell

Symplocos hartwegii A.DC.

Symplocos limoncillo Bonpl.

Symplocos tacanensis Lundell

\section{Theaceae}

Cleyera theoides (Sw.) Choisy

Freziera candicans Tul.

Freziera guatemalensis (Donn.Sm.) Kobuski

Symplococarpon flavifolium Lundell

Symplococarpon purpusii (Brandegee) Kobuski

Ternstroemia lineata DC. subsp. chalicophila (Loes.)

B.M.Barthol.

Ternstroemia oocarpa (Rose) Melch.

Ternstroemia tepezapote Schltdl. \& Cham.

\section{Thymelaeaceae}

Daphnopsis flavida Lundell

\section{Tiliaceae}

Triumfetta matudae Lundell

\section{Ulmaceae}

Ulmus mexicana (Liebm.) Planch.
EN

$\mathrm{EN}$

VU

CR

NT

EN

EN

VU

EN

VU

NT

EN

EN

EN
$\mathrm{Ab}$

$\mathrm{Ab}$

$\mathrm{Ab}$

$\mathrm{Ab}$

$\mathrm{Ab}$

$\mathrm{Ab}$

$\mathrm{Ab}$

$\mathrm{Ab}$

$\mathrm{Ab}$

$\mathrm{Ab}$

$\mathrm{Ab}$

$\mathrm{Ab}$

$\mathrm{Ab}$

H btp

Urticaceae

Coussapoa purpusii Standl. btc, btp

Ch

Ch

bc, bmm, bts

bmm

bq, bmm, vs

bmm

bc, bmm

bmm

bmm

bmm

bmm

bmm

bq, bmm, btp,

bts, vs

bc, bq, bmm, btp

$\mathrm{Ne}$

$\mathrm{Ne}$

$\mathrm{Ne}$

$\mathrm{Ne}$

$\mathrm{Ch}$

$\mathrm{Ne}$

Ch, H, O

$\mathrm{Ne}$

$\mathrm{Ne}$

Ch, G, S

he


Apéndice 1. Continuación

\begin{tabular}{|c|c|c|c|c|c|}
\hline Grupo/Familia/Especie & NOM & IUCN & HA & TV & DI \\
\hline \multicolumn{6}{|l|}{ Valerianaceae } \\
\hline Valeriana cucurbitifolia Standl. & & & $\mathrm{B}$ & bmm & $\mathrm{Ch}, \mathrm{O}$ \\
\hline \multicolumn{6}{|l|}{ Verbenaceae } \\
\hline Aegiphila skutchii Moldenke & & VU & $\mathrm{H}$ & bmm & $\mathrm{Ne}$ \\
\hline Aegiphila wigandioides Lundell & & & $\mathrm{H}$ & bmm & $\mathrm{Ch}$ \\
\hline \multicolumn{6}{|l|}{ Violaceae } \\
\hline Hybanthus chiapensis Lundell & & & $\mathrm{H}$ & bmm & $\mathrm{Ch}$ \\
\hline \multicolumn{6}{|l|}{ Viscaceae } \\
\hline Phoradendron ramosissimum Kuijt & & & $\mathrm{H}$ & bmm & $\operatorname{Tr}$ \\
\hline \multicolumn{6}{|l|}{ Winteraceae } \\
\hline Drimys granadensis L.f. var. mexicana (DC.) A.C.Sm. & & VU & $\mathrm{Ab}$ & bc, bmm & $\mathrm{Ne}$ \\
\hline
\end{tabular}

